\title{
Fluctuating selection and its (elusive) evolutionary consequences in a wild rodent population
}

\section{Abstract}

1 Temporal fluctuations in the strength and direction of selection are often proposed as 2 a mechanism that slows down evolution, both over geological and contemporary time3 scales. Both the prevalence of fluctuating selection and its relevance for evolutionary 4 dynamics remain poorly understood however, especially on contemporary time scales: 5 Unbiased empirical estimates of variation in selection are scarce, and the question of how 6 much of the variation in selection translates into variation in genetic change has largely 7 been ignored. Using long-term individual-based data for a wild rodent population, we 8 quantify the magnitude of fluctuating selection on body size. Subsequently, we estimate 9 the evolutionary dynamics of size, and test for a link between fluctuating selection and evolution. We show that, over the past 11 years, phenotypic selection on body size has fluctuated significantly. However, the strength and direction of genetic change have remained largely constant over the study period, i.e., the rate of genetic change was similar in years where selection favored heavier versus lighter individuals. This result suggests that over shorter timescales, fluctuating selection does not necessarily translate into fluctuating evolution. Importantly however, individual-based simulations show that the correlation between fluctuating selection and fluctuating evolution can be obscured by the effect of drift, and that substantially more data is required for a precise and accurate estimate of this correlation. We identify new challenges in measuring the coupling between selection and evolution, and provide methods and guidelines to overcome them. 


\section{${ }_{20}$ Introduction}

Selection, the causal covariation between trait values and fitness, shapes biodiversity in time and space and explains the general match between organisms and their environment (Darwin, 1859; Endler, 1986). Linking the sources of natural and sexual selection to the dynamics of evolution (defined here as a change in mean breeding value for the trait of interest) has been a major goal of evolutionary biology during the last century (e.g. Fisher, 1958), but for most of the 20th century progress has been hampered by the lack of a unified framework to quantify selection (Wade, 2006). This changed with the development of regression-based methods to measure the strength and direction of selection (Lande, 1979; Lande \& Arnold, 1983), which have enabled the estimation of selection gradients in a large variety of traits and biological systems (Kingsolver et al., 2001; Stinchcombe et al., 2008). This bonanza of estimates has shown that directional selection is stronger and more common than stabilizing selection, for both morphological and life-history traits (Kingsolver et al., 2001; Hereford et al., 2004; Hendry, 2017). At first sight, this pattern is contrary to expectations (Kingsolver \& Diamond, 2011): As most traits are heritable (Mousseau \& Roff, 1987; Postma, 2014), they are predicted to evolve towards their fitness optimum, with directional selection progressively being replaced by stabilizing selection. In practice, however, most traits evolve only very slowly and within a limited phenotypic range (Hendry \& Kinnison, 1999; Merilä et al., 2001; Brookfield, 2016).

One explanation for this paradox is that fitness landscapes are not constant over time, and populations are evolving towards a continuously changing fitness optimum (Fisher \& Ford, 1947; Lande, 1976). Whereas at any point in time directional selection may be strong, average selection gradients may be weaker, and if selection fluctuates not only in strength but also in direction, average selection may even be zero (Fig. 1 (A-C)). Fluctuating selection may thus slow down longer-term evolutionary adaptation, or even bring it to a halt (Jones et al., 2004; Estes \& Arnold, 2007), and it thereby constitutes an appealing explanation for the commonly observed lack of evolutionary change, i.e. evolutionary stasis, as well as for the commonness of directional selection (Merilä et al., 2001; Robinson et al., 2008; Bell, 2010). However, although fluctuating 
selection as an explanation for "macro-evolutionary" stasis is gaining theoretical and empirical support (Uyeda et al., 2011; Estes \& Arnold, 2007; Voje et al., 2015), our understanding of the importance of fluctuations in selection in shaping the evolutionary dynamics of natural populations on a much shorter time scale, e.g. from one generation to the next, is still limited. A few robust examples of temporal variation in selection exist (e.g., Grant \& Grant, 2002; Husby et al., 2011; Bergland et al., 2014; Milesi et al., 2016), but an assessment of the general micro-evolutionary relevance of fluctuating selection is hampered by the lack of a clear answer to two questions: (i) Does phenotypic selection commonly fluctuate, in strength and/or direction? (Hendry, 2017, pp.47-51) (ii) And if it does, do these fluctuations translate into fluctuations, in speed and/or direction, of genetic change?

The first question seemingly received a positive answer with the publication of a synthetic review of temporal replicates of selection from 89 studies, which concluded that phenotypic selection does indeed vary and reverse its direction among years (Siepielski et al., 2009). However, Morrissey \& Hadfield (2012) showed that most of these fluctuations can be ascribed to sampling variation, that is, the stochasticity that causes the realized value of a parameter to differ from the parameter of the data-generating process in finite populations. When sampling variation is accounted for, directional selection is in fact remarkably constant over time, both in magnitude and direction: Instead of estimating the variance of the distribution of temporal estimates of selection, as in Siepielski et al. (2009), tests for fluctuating selection must estimate the variance of the temporal distribution of selection (Morrissey \& Hadfield, 2012). As of yet, Chevin et al. (2015) are among the few to have done this: Using random regression mixed models which explicitly estimate the variance in selection gradients, they found that phenotypic selection on laying date fluctuated over a short time period in a population of great tits (Parus major Linnaeus, 1758). The generality of this finding however needs to be confirmed across a wider range of species, populations and traits, using the same, statistically robust, approach.

In addition to showing statistically significant variation in selection, two more points must be addressed to assess the evolutionary relevance of fluctuating selection. First, 
the precise pattern of fluctuation matters: Even in the presence of fluctuating selection, evolution will only come to a halt if the direction of selection changes regularly and the mean selection differential equals zero (Blanckenhorn, 2000; Hunt et al., 2004; Morrissey \& Hadfield, 2012) (see Fig. 1 (B)). Second, phenotypic selection, when defined as a nonzero phenotypic covariance between a trait and relative fitness, does not necessarily lead to an evolutionary response (see Fig. 1 (D)). The breeder's equation assumes that fitness covaries with phenotypic variation blindly, and does not distinguish between whether this phenotypic variation is the result of genetic or non-genetic variation (Rausher, 1992). When this assumption is violated and apparent selection is disproportionately dominated by an environmental covariance between the trait of interest and fitness, estimates of phenotypic selection provide a poor predictor of genetic change (Price \& Liou, 1989; Rausher, 1992; Morrissey et al., 2010; Bonnet et al., 2017). For instance, random infection of some individuals by a parasite may simultaneously drive among-individual variation in mass and variation in fitness, generating a covariation between mass and fitness, without mass causally affecting fitness.

While the latter is one of the general explanations for apparent evolutionary stasis, it is particularly relevant within the context of fluctuating selection: As fluctuating selection is often thought to be driven by environmental fluctuations (Bell, 2010; Chevin \& Haller, 2014), these may disproportionately shape (fluctuations in) the environmental component of selection. Fluctuations in the additive genetic covariance between the trait and fitness, i.e. in fluctuating evolution (Robertson, 1966; Price, 1970; Morrissey et al., 2012), can result from fluctuating selection only if the fluctuations involve the causal effects of the focal trait on fitness.

Here we take advantage of the eleven-year long monitoring of a population of snow voles (Chionomys nivalis Martins, 1842) to i) quantify fluctuating selection on body size, ii) describe the temporal dynamics of evolution in size, and iii) quantify the relationship between fluctuating selection and evolution. To this end, we first estimate directional selection on a year-to-year basis to quantify the variation in selection estimates. We then explicitly model these fluctuations of directional selection within a mixed model in order to 
account for sampling variance. Based on the sign of annual selection estimates, as well as on the ratio of the variance in selection over the mean strength of selection, we also assess the probability of selection reversal. These analyses are performed for total selection, as well as for fertility and viability selection separately. Second, we use a quantitative genetic framework to describe the general pattern of evolution over the study period, and estimate the rate of evolution of size on a year-to-year basis. Third, we combine analyses of selection and estimates of evolutionary change to assess the coupling between variation in the strength and sign of selection and evolution. Finally, we perform a series of individual-based simulations to infer the statistical power of our test for fluctuating selection and its evolutionary relevance, which is crucial when it comes to interpreting any negative results.

\section{Material and methods}

\section{Study population}

From 2006 to 2016, a wild population of snow voles (Chionomys nivalis Martins, 1842) has been monitored intensively. This population, which consists of 80-230 individuals (table 1), is located in the Swiss Alps, near Chur (N46 $48^{\prime}, \mathrm{E}^{\circ} 34^{\prime} ; 2,030$ m.a.s.l.). The study area consists of 5 ha of scree with sparse vegetation, surrounded by meadows, forest and a steep cliff. Because the snow vole shows an overwhelming preference for rocky environments (Janeau \& Aulagnier, 1997; Luque-larena et al., 2002), the monitored population is ecologically fairly isolated. Nevertheless, it receives on average 8.6 immigrants per year (table 1, see also García-Navas et al., 2015).

Snow voles are live-trapped during two to five trapping sessions taking place between late May and early October. To this end, the study area is overlaid with a $10 \times 10 \mathrm{~m}$ grid consisting of a total of 559 cells with stable geographic coordinates. A trapping session consists of four trapping nights, necessary to cover all four quarters of the study area. During each trapping session, a Longworth trap (catch-and-release trap, Penlon Ltd, Oxford, UK) filled with hay and baited with apple, hamster food and peanut butter 
is placed in every cell. Individuals captured for the first time are ear-clipped (2mm diameter, thumb type punch, Harvard Apparatus, Massachusetts, USA) and individually marked with a subcutaneous PIT tag (ISO transponder, Tierchip Dasmann, Tecklenburg, Germany). Ear-clips are preserved in 95\% ethanol + 5\% TE. For each capture, we record individual identity, geographic coordinates, body mass, body length, tail length, sex and age.

Ear clips are stored at $-20^{\circ} \mathrm{C}$ until DNA extraction. All individuals are genotyped for 18 autosomal microsatellites using snow vole-specific primers (Wandeler et al., 2008; García-Navas et al., 2015). In addition, the sex of all individuals is confirmed by sequencing the Sry locus (Gubbay et al., 1990; Wandeler et al., 2008). Finally, the mitochondrial control region is sequenced, and all males are genotyped for one Y-linked microsatellite and three Y-linked insertion-deletions (Wandeler \& Camenisch, 2011). Based on the autosomal microsatellite genotypes, we reconstruct the pedigree of the population using the maximum likelihood-based program COLONY (Wang, 2004; Jones \& Wang, 2010) and the Bayesian R package MasterBayes (Hadfield et al., 2006; R Core Team, 2015). The pedigree is then checked for consistency using the Y-linked markers and the mitochondrial haplotypes. This procedure allows the identification of most of the parental links (91\%) as well as the identification of likely immigrants (individuals first captured as adults and with two unknown parents).This well-resolved pedigree is used to define annual and lifetime reproductive success, as well as to estimate the relatedness among all pairs of individuals.

A mark-recapture analysis has shown that between-session recapture probabilities are very high (adults: $92.4 \% \pm 1.1$; juveniles: $81.1 \% \pm 3.0$ ). Therefore, the between-year recapture probability is effectively 1 , and the non-capture of an individual in a given year can be directly equated with death or permanent emigration without the need for mark-recapture modeling.

\section{Fitness measures}

We considered three measures of fitness: (i) survival from one year to the next, $\phi_{i, t}$, based on whether an individual $i$ observed in year $t$ is observed again in year $t+1\left(\phi_{i, t}=1\right)$ or 
not $\left(\phi_{i, t}=0\right)$; (ii) annual reproductive success, $\rho_{i, t+1}$, the number of juveniles born from $i$ during the year $t+1$, that is, after $i$ survive to the next year (but irrespective of juvenile survival); (iii) an annualized measure of overall fitness, similar to that used in Qvarnström et al. (2006), $F_{i, t}=2 \phi_{i, t}+\rho_{i, t+1} . F_{i, t}$ is an appropriate measure of fitness in the context of studying evolution with overlapping generations because it captures the production of all the individuals present in year $t+1$ by all the individuals present in year $t$ (Fig. 2). In our measure of total fitness $\left(F_{i, t}\right)$, survival is multiplied by two because an individual is twice as related with itself as with its offspring. The alternative of dividing the number of offspring by two would result in non-integer numbers, which cannot be fitted using standard generalized linear models.

Note that the distribution of this annualized measure of fitness $F$ will never exactly comply with a Poisson distribution because 1 is an impossible value (an individual must survive, thus obtaining 2 fitness points, before it can reproduce). However, computer simulations (described in SI 1) confirm that selection measured as the covariance between a trait and $F$ perfectly predicts genetic change from $t$ to $t+1$ when the heritability of the trait equals one (here and in the rest of the manuscript, heritability refers to the additive genetic heritability, i.e., narrow-sense heritability). Moreover, with a trait heritability smaller than one, the slope of evolution on selection was equal to the simulated heritability (see SI 1), as predicted by the breeder's equation.

\section{Measuring body size}

Although our aim is to gain a better understanding of the evolutionary relevance of fluctuating selection on body size, the use of absolute body mass measurements is complicated by the fact that juvenile age is unknown. As we have shown previously, not correcting for this age-related variation in body mass provides a misleading description of the causal relationship between body size and fitness, and it provides a poor prediction of adaptive evolution in this system (Bonnet et al., 2017).

Rather than using growth curves to account for age-related variation in body mass (see Bonnet et al., 2017), here we chose to instead divide body mass by body length to obtain 
a body mass index (BMI). We used mass over length rather than length squared, a more common body mass index, because the distribution of the former was close to a Gaussian distribution, while the latter was right-skewed. BMI is more repeatable than body mass in juveniles (0.73 versus 0.62 ). In order to obtain standardized selection gradients, we standardized BMI across all years by subtracting the mean and dividing by its standard deviation. An overview of the sample sizes is given in table 1.

\section{Selection analysis}

Selection was estimated with a series of generalized linear models (GLMs) and generalized linear mixed models (GLMMs), regressing fitness measures on BMI. Mixed models were fitted with the R-package MCMCglmm (Hadfield, 2010). This package accounts for overdispersion when modeling Poisson traits

Using the annualized measure of overall fitness, $F_{i, t}$, we first estimated selection on a year-by-year basis using a Poisson GLM with a log link, where the expected fitness of individual $i$ at time $t$ is predicted from:

$$
\log \left(F_{i, t}\right)=\mu_{F, t}+\beta_{F, a, t} a_{i, t}+\beta_{F, s, t} s_{i}+\beta_{F, a s, t} a s_{i, t}+\left(\beta_{F, z, t}\right) z_{i, t},
$$

where $a_{i, t}$ is the age (juvenile or adult) of individual $i$ at year $t, s_{i}$ is the sex of $i, z_{i, t}$ is the phenotype (BMI) of $i$ at $t, \mu_{F, t}$ is the intercept of the regression, $\beta_{F, a, t}$ is the effect of age, $\beta_{F, s, t}$ is the effect of sex, $\beta_{F, a s, t}$ is the interaction sex-by-age, and $\beta_{F, z, t}$ is the strength of selection on BMI. Because we used a log link, $\beta_{F, z, t}$ is a selection gradient sensu Lande \& Arnold (1983) (Smouse et al., 1999; Firth et al., 2015).

The standard deviation in the yearly estimates of selection $\left(\operatorname{SD}\left(\hat{\beta}_{F, z, t}\right)\right)$ gives a first idea about the temporal dynamic of selection, but as it includes sampling variance, it will always overestimate the real variation in selection (Morrissey \& Hadfield, 2012).

Second, we estimated overall selection by fitting a Poisson GLM to pooled data from 
all the years, without taking into account temporal variation:

$$
\log \left(F_{i, t}\right)=\mu_{F}+\beta_{F, a} a_{i, t}+\beta_{F, s} s_{i}+\beta_{F, a s} a s_{i, t}+\beta_{F, z} z_{i, t} .
$$

Third, we directly estimated variation in selection by fitting a random regression to the full dataset. Thus, we expanded model (2) to a Poisson GLMM by including a random intercept and a random slope for fitness as a function of BMI:

$$
\log \left(F_{i, t}\right)=\mu_{F 0}+\mu_{F, t}+\beta_{F, a}^{\prime} a_{i, t}+\beta_{F, s}^{\prime} s_{i}+\beta_{F, a s}^{\prime} a s_{i t}+\left(\beta_{F, z}^{\prime}+\zeta_{F, t}\right) z_{i, t}
$$

where $\beta_{F, z}^{\prime}$ is the median selection estimate, $\mu_{F, t}$ is the random deviation of the global intercept $\left(\mu_{F 0}\right)$ in year $t$ and $\zeta_{F, t}$ is the deviation of selection (i.e. the slope) in year $t$. The random effects $\mu_{F, t}$ and $\zeta_{t}$ are assumed to be mutivariate normal with variances $\sigma_{F, \mu}^{2}$ and $\sigma_{F, \zeta}^{2}$, and a covariance $\sigma_{F,(\mu, \zeta)}$. The main parameter of interest in this equation is $\sigma_{F, \zeta}^{2}$, which captures temporal variation in selection and is free of sampling variance (Chevin et al., 2015).

The median selection gradient estimate $\left(\beta_{F, z}^{\prime}\right)$ from model (3) differs from the estimate across all years $\left(\beta_{F, z}\right)$ from model (2) if the estimate of $\sigma_{F, \zeta}^{2}$ is different from 0 and data are not perfectly balanced among years. Whereas the latter, $\beta_{F, z}$, is the best estimate of the overall selection, the former, $\beta_{F, z}^{\prime}$, is the selection occurring in a "standard" year. The ratio of $\sigma_{F, \zeta} /\left|\beta_{F, z}^{\prime}\right|$ provides an indication of the likelihood of a reversal in the direction of selection. Assuming that the annual selection gradients follow a Gaussian distribution (as the random regression assumes), this ratio is similar to an inverse $Z$-value. Values around 0.6 indicate rare reversals (5\% of the time), and values above 2 indicate common reversals (more than $31 \%$ of the time).

We repeated these analyzes for annual reproductive success $(\rho)$, again using a Poisson GLMM, and for over-winter survival $(\phi)$, using a logistic regression (i.e., a categorical GLMM with two levels). As was the case for $F$, because we use a log link, estimates of the strength of selection using $\rho$ are selection gradients sensu (Lande \& Arnold, 1983) (Smouse et al., 1999; Firth et al., 2015). Although this is not the case for $\phi$, the sign 
and strength of estimates of selection are still interpretable qualitatively. For Fig. 3C, we back-transformed the selection gradients to the scale of the data, following Janzen \& Stern (1998). We did not back-transformation the selection parameters in table 2 because it is unclear how to convert the variance in the slope to the scale of the data. The main parameters of interest, the variances in the slope of selection, are written $\sigma_{\phi, \zeta}^{2}$ and $\sigma_{\rho, \zeta}^{2}$, for viability and fertility, respectively.

Finally, we refitted model (3) with BMI standardized within years (subtracting the average and dividing by the standard deviation for each year), in order to evaluate whether the variation in selection comes from changes in phenotypic variance (resulting in a lower estimate of $\sigma_{F, \zeta}$ ), or rather from a moving fitness landscape (in which case $\sigma_{F, \zeta}$ would be unaffected).

\section{Expected correlation between selection and evolution}

We used individual-based computer simulations to explore the expected relationship between selection and evolution in a population similar to the snow vole population. Building on the model developed in Bonnet \& Postma (2016), we simulated a heritable phenotypic trait, as well as reproductive success and survival from one year to the next, in discrete time, and considered two age-classes (non-reproductive juveniles, and reproductive adults). For details of the simulation algorithm, see SI 1.

For every simulation replicate, we extracted the annual selection (standardized selection differentials) and annual evolution (change in mean breeding value for all individuals alive in one year and all individuals alive in the next year), and computed the correlation between them. We repeated this 500 times to estimate the expected distribution of the correlation given a set of parameter values. We tested the exactness of the simulation algorithm by comparing the ratio of evolution $(R)$ over selection $(S)$ to the heritability simulated $\left(h^{2}\right)$ (SI 1). Besides, the equality $\frac{R}{S}=h^{2}$ holds when the assumptions of the breeder's equation are met, and our simulations provide a null-model against which to test our hypothesis that the assumptions of breeder's equation are not valid in the snow vole population (Bonnet et al., 2017). 
In a first step, we parameterized the simulations to closely match important properties of the snow vole dataset: sample size in every year; amount of genetic and environmental variance in size; strength and variability of selection. In a second step, we varied the heritability (while holding the phenotypic variance constant) or the monitoring duration, which allowed us to explore how these two parameters affect the correlation between selection and evolution (see Fig. 5 and SI 1.2).

\section{Inference of evolution and the contribution of fluctuating selec- tion}

We estimated all quantitative genetic parameters by fitting animal models (Henderson, 1950, 1975, 1976; Kruuk, 2004) using MCMCglmm. This Bayesian package allows extracting and combining full posterior distributions of parameters. Unless stated otherwise, all calculations were done on the posterior distributions (rather than on point estimates) in order to propagate estimation uncertainty and account for covariation between parameters. For all models, we ran a MCMC chain long enough to obtain 1,000 posterior samples, with a thinning interval large enough to keep the autocorrelation for any parameter below $10 \%$, and added a burnin of about $20 \%$ of the total iterations. For fixed effects we always used the default priors, which are normal distributions with means of zero and variances of $10^{1} 0$. For random effects, we used inverse-Wishart priors, with parameters $V=1$ and $n u=0.002$ for univariate models (equivalent to an inverse gamma distribution with parameters $\alpha=\beta=0.001$ ), and with $V=\boldsymbol{I}(n)$ and $n u=n+1$, where $n$ is the number of traits considered and $\boldsymbol{I}(\boldsymbol{n})$ is an identity matrix of dimension $n$, for multivariate models (see SI 2for visual representation). All animal models included genetic groups (Quaas, 1988) to model gene flow from immigrants and to account for a difference in BMI breeding values between immigrants and the base population of the pedigree (Hadfield et al., 2010; Wolak \& Reid, 2017). To this end, we considered two groups, the base population and immigrants, and used the explicit fixed effect specification following Wolak \& Reid (2017). Because additive genetic variation in fitness is a prerequisite for a response to selection, we first estimated the genetic variance in our fitness proxy $F$, using a univariate animal 
model assuming a Poisson distribution with a log link. Overdispersion is accounted for by default in MCMCglmm. The model included an intercept, age, sex and their interaction, as well as date of capture as fixed effects, and additive genetic effects, individual identity (i.e. permanent environment effects), maternal identity and year as random effects. Additive genetic variance and heritability were estimated after transformation from the latent scale to the data scale, by integrating over all the random effects and fixed effects (Morrissey, 2015; de Villemereuil et al., 2016), using the R package QGglmm (de Villemereuil et al., 2016)

We then used two approaches to infer the yearly rates of evolution in BMI: 1) a univariate approach based on BLUPs regression (Henderson, 1950; Hadfield et al., 2010) and 2) a multivariate approach based on the Robertson-Price identity (Price, 1970; Morrissey et al., 2012; Bonnet et al., 2017).

For the first approach, we fitted a univariate animal model to BMI data, including age, sex, their interaction, as well as date of capture as fixed effects, and random additive genetic, permanent environment (i.e. individual identity), maternal (maternal identity) and year effects. For every two successive years, we computed the genetic change in BMI between the two sets of living individuals using best linear unbiased predictors (BLUPs) for breeding values (following Hadfield et al., 2010). We simulated genetic drift down the pedigree of the snow vole population (following Hadfield et al., 2010, and using the function $\mathrm{rbv}()$ in MCMCglmm, with genetic groups to account for immigration), and computed the range of genetic change between years that genetic drift can produce. We visualized the temporal dynamics of genetic evolution of BMI by fitting a time spline (i.e. a smoother) to the breeding values of all individuals alive in each year. The spline was fitted using a generalized additive model in the R package mgcv (Wood, 2011). We estimated a time spline for each posterior sample of the distributions of individual breeding values, in order to obtain the posterior distribution of evolution. We tested for the significance of evolution using the same approach but using linear regressions.

To quantify the role of variation in selection in shaping the population's evolutionary trajectory, we computed the correlations between the annual estimates of selection 
gradients and the change in breeding values to the next year. We used the posterior distribution of changes in breeding values, but only the point estimate of annual selection gradients, to obtain a posterior distribution of correlations.

For the second approach, we would ideally have estimated the genetic and environmental selection gradients for every year by fitting a multivariate animal model treating BMI in each year as a different trait. However, although we did initially fit such a model, because of data limitations it did not reach convergence and the priors dominated the posterior distribution. Instead we split the data in two groups of years: those where our estimates of selection (as estimated above) were positive, and those where they were negative (see Reed et al., 2016 for a similar approach). We considered BMI in these two groups of years as two different traits $\left(M_{+}\right.$and $M_{-}$, respectively). We subsequently fitted a trivariate animal model to the two BMI traits and our annualized measure of fitness $(F)$. This model allows the estimation of an additive genetic covariance between BMI and fitness for the two year classes. Based on the Robertson-Price equation, these covariances provide a direct and unbiased expectation of the rate of evolution during the two groups of years (Robertson, 1966; Price, 1970, 1972; Frank, 2012; Morrissey et al., 2012). By measuring fitness on a yearly basis we removed the assumption of non-overlapping generations. We compare and explain the advantages and drawbacks of both approaches in the discussion.

The trivariate animal model can be written as

$$
\left[M_{+}, M_{-}, F\right] \sim b X+Z_{1} a+Z_{2} m+Z_{3} p+Z_{4} y+I r
$$

where $\boldsymbol{X}, \boldsymbol{Z}_{\mathbf{1}}, \boldsymbol{Z}_{\mathbf{2}}, \boldsymbol{Z}_{\mathbf{3}}$ and $\boldsymbol{Z}_{\mathbf{4}}$ are design matrices relating BMI and fitness observations to the parameters to estimate, $\boldsymbol{b}$ is a matrix of fixed effects, $\boldsymbol{a}, \boldsymbol{m}, \boldsymbol{p}$ and $\boldsymbol{y}$ are random effects accounting for the variance associated with additive genetic, maternal, permanent environment and year effects, respectively. Residuals $\boldsymbol{r}$ are assumed to be normally distributed and independent, and are therefore associated to observations by an identity matrix $\boldsymbol{I}$. The fixed part of the model matches that used for each trait in univariate models (see above). 
The matrix of breeding values $\boldsymbol{a}$ follows a multivariate normal distribution

$$
\boldsymbol{a} \sim M V N(\mathbf{0}, \boldsymbol{A} \otimes \boldsymbol{G})
$$

where $\boldsymbol{A}$ is the relatedness matrix between all individuals, and $\boldsymbol{G}$ is the additive genetic variance covariance matrix between the three traits.

$$
\boldsymbol{G}=\left(\begin{array}{ccc}
\sigma_{A}^{2}\left(M_{+}\right) & \sigma_{A}\left(M_{+} M_{-}\right) & \sigma_{A}\left(M_{+} F\right) \\
\sigma_{A}\left(M_{+} M_{-}\right) & \sigma_{A}^{2}\left(M_{-}\right) & \sigma_{A}\left(M_{-} F\right) \\
\sigma_{A}\left(M_{+} F\right) & \sigma_{A}\left(M_{-} F\right) & \sigma_{A}^{2}(F)
\end{array}\right)
$$

where $\sigma_{A}^{2}\left(M_{+}\right)$and $\sigma_{A}^{2}\left(M_{-}\right)$are the additive genetic variance for BMI in years with positive selection and negative selection, respectively, $\sigma_{A}\left(M_{+} M_{-}\right)$is the additive genetic covariance between BMI in the two group of years, $\sigma_{A}^{2}(F)$ is the additive genetic variance in fitness across years, i.e. the genetic differential of fitness itself (Fisher, 1958), and finally, $\sigma_{A}\left(M_{+} F\right)$ and $\sigma_{A}\left(M_{-} F\right)$ are the additive genetic covariances between fitness and BMI in years with high and low selection, respectively. We computed the genetic gradients for both groups of years as $\beta_{A+}=\sigma_{A}\left(M_{+} F\right) / \sigma_{A}^{2}\left(M_{+}\right)$and $\beta_{A-}=\sigma_{A}\left(M_{-} F\right) / \sigma_{A}^{2}\left(M_{-}\right)$. The additive genetic correlation between BMI in the two groups of years was computed as $\sigma_{A}\left(M_{+} M_{-}\right) / \sigma_{A}\left(M_{+}\right) \sigma_{A}\left(M_{-}\right)$.

Environmental selection differentials $\sigma_{E}\left(M_{+} F\right)$ and $\sigma_{E}\left(M_{-} F\right)$ were calculated as the sum of the covariances between BMI and fitness in the random effect variance-covariance matrices for permanent environment, maternal identity and the residuals. The environmental variances $\sigma_{E}^{2}\left(M_{+} F\right)$ and $\sigma_{E}^{2}\left(M_{-} F\right)$ were obtained by summing the variance components of the same random effects. Subsequently, the environmental selection gradients were obtained using $\beta_{E+}=\sigma_{E}\left(M_{+} F\right) / \sigma_{E}^{2}\left(M_{+}\right)$and $\beta_{E-}=\sigma_{E}\left(M_{-} F\right) / \sigma_{E}^{2}\left(M_{-}\right)$. Finally, the phenotypic selection gradients were recovered using $\left(\sigma_{A}\left(M_{+} F\right)+\sigma_{E}\left(M_{+} F\right)\right) /\left(\sigma_{A}^{2}\left(M_{+}\right)+\right.$ $\left.\sigma_{E}^{2}\left(M_{+}\right)\right)$and $\left(\sigma_{A}\left(M_{-} F\right)+\sigma_{E}\left(M_{-} F\right)\right) /\left(\sigma_{A}^{2}\left(M_{-}\right)+\sigma_{E}^{2}\left(M_{-}\right)\right)$. The phenotypic selection gradients represent selection on the phenotype sensu Lande \& Arnold (1983), while the environmental and the additive genetic selection gradient represent the indirect action 
of selection (they are not selection in a strict sense) on the environmental and additive genetic part of phenotypic variation, respectively. These three gradients are equal if the assumptions of the breeder's equation are met, i.e. when the phenotypic covariation between the trait and fitness is causal and not in part the result of unmeasured environmental covariates (Rausher, 1992). For size-related traits, disproportionately large environmental selection gradients might be interpreted as the effect of non-heritable body condition shaping both mass and fitness, whereas the additive genetic selection gradient captures causal, direct selection on the trait.

To confirm the values of the genetic and environmental covariances between BMI and $F$, we additionally ran two bivariate animal models with $M_{+}$and $F$, and $M_{-}$and $F$, respectively. In addition, to confirm the stability of the genetic covariance through time (see Results), we re-fitted the trivariate model, but instead of distinguishing between years with positive and negative phenotypic selection, we treated BMI in every second year as one trait (equivalent to $M_{-}$), and BMI in the other years as another trait (equivalent to $\left.M_{+}\right)$. Finally, we assessed potential biases in $\beta_{A}-\beta_{E}$ generated by splitting the dataset into two groups of years on the basis of the direction of phenotypic selection: Using the R-package pedantics (Morrissey \& Wilson, 2010), we simulated phenotypes and fitness values with $\beta_{A}$ expected to be equal to $\beta_{E}$, split the data based on the sign of the selection differential in every year, and fitted animal models to estimate the expected distribution of $\beta_{A+}-\beta_{E+}$ and $\beta_{A_{-}}-\beta_{E-}$. See SI 4for details.

\section{Results}

\section{Yearly estimates of selection}

Annual selection gradients varied considerably (standard deviation $=1.198$ ) around the overall selection gradient $(0.639 \pm \mathrm{SE} 0.18$; Fig. $3(\mathrm{~A}))$. Estimates of total selection were mostly positive, but appeared to have been negative in three years. Although the standard deviation of the yearly estimates was greater than the overall selection gradient, a large proportion of this variation must be attributable to sampling error. Indeed, yearly 
selection was estimated with much less precision than overall selection, as is reflected by a mean standard error of the yearly estimates of 0.753 . Fertility and viability selection gradients showed similar patterns (Fig. 3 (B-C)): The standard deviations of the estimates of viability and fertility selection were high, but so were the mean standard errors of these estimates (table 2)

\section{Fluctuation of selection}

Fitting equation (3), we estimated $\sigma_{F, \zeta}=0.691(95 \% \mathrm{CI}[0.461 ; 1.153])$ and $\sigma_{F, \zeta} /\left|\beta_{F, z}^{\prime}\right|=$ 1.156. Assuming a normal distribution of selection gradients and a ratio of standard deviation over mean of 1.156, a reversal of the direction of selection would be occasional (about once every 5 years). Refitting equation (3) with BMI standardized within years yielded a similar estimate of $\sigma_{F, \zeta}(0.674[0.433 ; 1.162])$.

Variance in fertility selection was estimated as $\sigma_{\rho, \zeta}=0.512(95 \% \mathrm{CI}[0.385 ; 0.779])$, more than twice the absolute median selection gradient (table 2), meaning that selection was very likely to change direction. Variance in viability selection was estimated as $\sigma_{\phi, \zeta}=0.642(95 \% \mathrm{CI}[0.409 ; 1.024])$

The correlations between random intercepts and random slopes were close to zero for all three models $\left(\sigma_{F,(\mu, \zeta)}=-0.11, \sigma_{\rho,(\mu, \zeta)}=0.08, \sigma_{\phi,(\mu, \zeta)}=-0.16\right)$, suggesting appropriate estimation of the variance components (the correlation is close to 1 or -1 when the model fit is (quasi-)singular).

\section{Fluctuation of evolution}

There was a small but significant amount of additive genetic variation in our proxy of annual fitness: On the latent scale of the Poisson model, the additive genetic variation was estimated to be $0.299[0.086 ; 0.692]$. On the scale of the data, this translates into an additive genetic variance of $0.280[0.001 ; 0.994]$ and a heritability of $1.13 \%[0.06 \% ; 5.01 \%]$. This is comparable to the heritability of lifetime fitness in Bonnet et al. (2017), which used a lifetime rather than annual measure of fitness.

We found significant additive genetic variation in BMI $\left(167 \mathrm{~g}^{2} / \mathrm{m}^{2}[98 ; 307]\right.$; heritabil- 
ity $=16.7 \%[8.9 \% ; 26.3 \%])$. In this population there is evidence for adaptive evolution towards lower body size from 2006 to 2014 (Bonnet et al., 2017). This pattern is also found for BMI (Fig. 4), with a decrease in mean breeding value of $-3.69[-8.13 ; 0.51]$ on a mean trait value of 288 , and a $3 \%$ probability that the trend is not negative. The trend might have reversed between 2014 and 2016 (Fig. 4), when there is some evidence that the breeding values for BMI have increased by $2.51[-1.1 ; 8.42](6.5 \%$ probability that the change is not positive). The decrease in breeding values from 2006 to 2014 is unlikely to have been produced solely by genetic drift, with a probability that drift generated a decrease that is at least as large of $p_{\mathrm{MCMC}}=0.064$ (see also Bonnet et al., 2017), whereas drift could have produced the rebound from 2014 to $2016\left(p_{\mathrm{MCMC}}=0.24\right)$.

\section{From selection to evolution}

Given the heritability of BMI and the duration of the snow vole monitoring, the correlation between selection gradients and change in breeding values from one year to the next is expected to be strongly positive on average, but also highly variable: individual-based simulations show that the distribution of the correlation between selection and evolution has its mode at 0.68 with $95 \%$ CI $[-0.12 ; 0.94]$ (see Fig. 5 and SI 1.2). This variability is due to strong genetic drift combined with the relatively small number of years. Increasing the heritability of the trait increases the expected correlation and reduces its variability (Fig. 5), while increasing the duration of the monitoring reduces variability only (SI 1.2).

Empirically, the correlation is estimated with a lot of uncertainty and is not statistically significantly different from zero $\left(p_{\mathrm{MCMC}}=0.08\right)$. Nevertheless, the most likely value is positive (mode $0.33,95 \% \mathrm{CI}[-0.07 ; 0.71]$ ) and does not lie in the extreme tail of the theoretical distribution (Fig. 5).

As expected, in years with positive selection (based on selection gradients from yearby-year GLMs, see above), the selection gradient reconstructed from our trivariate animal model was positive, while it was negative for years with negative selection gradients (Fig. 6). Importantly however, the genetic gradients were negative in both groups of years (Fig. 6) and did not differ from each other $\left(\beta_{A_{+}}-\beta_{A_{-}}=-0.0011,95 \% \mathrm{CI}[-0.0164 ; 0.0112]\right.$, 
$\left.p_{\mathrm{MCMC}}=0.72\right)$.

The environmental gradients, on the other hand, differed from each other $\left(\beta_{E+}-\beta_{E-}=\right.$ 0.0218, 95\% CI $\left.[0.0009 ; 0.0355], p_{\mathrm{MCMC}}=0.036\right)$, with $\beta_{E+}$ being significantly positive, and $\beta_{E-}$ slightly negative. Moreover, during years of positive selection, the genetic and environmental gradients were of opposite sign (Fig. 6), and significantly different $\left(\beta_{A+}-\right.$ $\left.\beta_{E+}=-0.0260,95 \% \mathrm{CI}[-0.0454 ;-0.0028], p_{\mathrm{MCMC}}=0.034\right)$. On the other hand, during years of negative selection, the genetic and environmental gradients were both negative (Fig. 6), and not significantly different $\left(\beta_{A_{-}}-\beta_{E-}=-0.0045,95 \% \mathrm{CI}[-0.0282 ; 0.0205]\right.$, $\left.p_{\mathrm{MCMC}}=0.824\right)$. Finally, the genetic correlation between BMI in positive selection years and BMI in negative selection years was strongly positive $(0.61,95 \% \mathrm{CI}[0.22 ; 0.83])$. The stability of these results was confirmed by splitting the data set differently (see SI 3).

\section{Discussion}

Here we have shown that selection on BMI fluctuates in a natural population of snow voles. In addition, we have shown that BMI has evolved, but that both the rate and direction of evolution do not appear to be tightly coupled with the dynamics of selection. Below we discuss the methodological challenges posed by the quantification of variation in selection and its evolutionary relevance, and our contribution to their resolution. We then discuss whether our analyses can inform us about the mechanisms of fluctuating selection, and what is needed to answer the questions that are beyond the reach of our analyses. Finally, we discuss the importance of timescale when studying variation in selection and evolution.

\section{The modeling of evolution and selection}

The random regression method Chevin et al. (2015) provides a statistically rigorous way to quantify and test for the significance of variation in selection. On its own, however, a random regression does not address the evolutionary relevance of fluctuating selection. To establish the latter, two additional issues need to be investigated: (i) Variation in the 
strength of selection will reverse the direction of evolution only if it fluctuates not only in strength, but also in direction (see Fig. 1B and C); (ii) As selection does not always lead to an evolutionary response (Rausher, 1992; Merilä et al., 2001; Morrissey et al., 2010), fluctuating selection does not necessarily translate into fluctuating evolution (see Fig. 1D).

To address the first issue, we considered where the distribution of selection gradients, estimated by a random regression, is located relative to zero. If this distribution is centered around zero, selection reversal is frequent, whereas if the distribution does not overlap much with zero, selection reversal is rare. We evaluated the likelihood of selection reversal by calculating the ratio of the standard deviation of selection gradients over the absolute median selection gradient $\left(\sigma_{\zeta} /\left|\beta_{z}^{\prime}\right|\right)$. As this ratio increases, the fluctuation of selection becomes increasingly biologically relevant, and a reversal becomes increasingly likely. However, even if the distribution of selection gradients is symmetric (which it does not have to be), as our estimate of the distribution of selection gradients is based on a finite number of years, it is unlikely to comply with an inverse $Z$-distribution. Furthermore, as selection gradients may show temporal autocorrelation, the appropriate number of degrees of freedom is unclear. Furthermore, as selection gradients may show temporal autocorrelation, the appropriate number of degrees of freedom is unclear. Hence, we are reluctant to translate this ratio into a probability of reversal. Nevertheless, it gives a qualitative assessments of the likelihood of reversal that could be developed further into a more quantitatively rigorous measure.

To address the second issue, we estimated the coupling between variation in selection and variation in genetic change. This exercise proved to be challenging and provided somewhat mixed results. In a first approach, we computed the correlation between selection and year-to-year changes in breeding values by relating the full distribution of the change in BLUPs for breeding values to point estimates of selection gradients. Therefore, the uncertainty accompanying the selection estimates was not propagated to this correlation. In contrast, the trivariate animal model estimates selection and evolution within the same model, along with their respective uncertainties. This allows for the integration 
of uncertainty in both selection and evolution when comparing genetic and environmental gradients, and to take into account the non-independence of their posterior distributions. Unfortunately however, this multivariate approach is particularly data-hungry, and the snow vole population is too small to estimate year-specific genetic parameters. As a consequence, we were forced to compare groups of years with negative and positive selection, although this approach generates a bias in the estimated difference between genetic and environmental gradients (SI 4). Fortunately, in our particular case, the bias is in the direction opposite to our findings, and our analyses are hence statistically conservative. Nevertheless, the presence of biases makes this approach risky, and its correct interpretation relies on computationally intense simulations. In conclusion, whenever the population size allows for it, and to avoid the aforementioned problems and biases, we advocate the use of year-specific multivariate animal models for assessing the coupling of selection and evolution.

\section{Coupling of selection and evolution}

Simple algebra shows that a positive correlation between selection and evolution is expected. For a trait $z$, a selection gradient is the ratio of the phenotypic covariance between trait and relative fitness, over the phenotypic variance in the trait:

$$
\beta_{P}=\frac{\sigma_{P}(z, F)}{\sigma_{P}^{2}(z)} .
$$

Assuming a standard quantitative genetic model in which there is no correlation or interaction between the genetic effects and the environmental effects (i.e. an absence of genotype-environment correlations and interactions), $z$ can be decomposed into additive genetic effects and environmental effects $z=a+e$. Similarly, the phenotypic covariance $\left(\sigma_{P}(z, F)\right.$, i.e. the selection differential) can be decomposed into an additive genetic $\left(\sigma_{A}(z, F)\right)$ and an environmental covariance $\left(\sigma_{E}(z, F)\right)$. Therefore, the phenotypic selec- 
tion gradient $\left(\beta_{P}\right)$ can be written as:

$$
\beta_{P}=\frac{\sigma_{A}(z, F)+\sigma_{E}(z, F)}{\sigma_{P}^{2}(z)} .
$$

According to the Robertson-Price identity (Robertson, 1966; Price, 1970), $\sigma_{A}(z, F)$ is the expected rate of genetic change. From the above it follows that the phenotypic selection gradient is likely to be positively correlated with evolution (provided the latter is nonzero). Even if their signs are opposite in all years, years with more positive selection gradients will go with less negative genetic change, and vice versa.

Using computer simulations, we found that for our dataset, if the relationship between trait and fitness (i.e. selection) is causal (Reed et al., 2016), the correlation between evolution and selection is expected to be relatively strong and positive $(0.68,95 \% \mathrm{CI}$ $[-0.12 ; 0.94])$. Nevertheless, this correlation has a $7.8 \%$ (SE $0.2 \%)$ probability to be zero or negative because of the potentially large effect of genetic drift.

The observed correlation between selection and evolution among years was not significantly different from zero nor from the theoretical expectation (see Results). Nevertheless, there are good reasons to think that phenotypic selection on size may not translate into consistent evolution: 1) across-year selection favors larger sizes (Fig. 3), while evolution is towards smaller sizes (Fig. 4); and 2) in years of positive selection, the genetic gradient differs in sign from the environmental and phenotypic gradients (Fig. 6), a pattern also seen when analyzing all years together (Bonnet et al., 2017). Therefore, our finding that the correlation between selection and evolution does not deviate significantly from the null-expectation may be the result of a lack of statistical power, and not of the lack of an environmental bias.

\section{What drives fluctuations in selection?}

Although our random regression and quantitative genetic models give a thorough description of the dynamics of selection and evolution in this population, they do not provide direct insight into the underlying mechanisms. We have shown that selection fluctuates, 
and thus that the relationship between size and fitness changes at the population level, but why does selection change? Different processes may lead to the same distribution of directional selection gradients, and based on the analysis of selection gradients alone it is difficult to distinguish fluctuations due to a moving fitness optimum from those due to a change in the distribution of phenotypes among years (Chevin \& Haller, 2014). The latter could have played a role here as we find substantial variation between years in both the mean phenotype (ranging between $277 \mathrm{~g} / \mathrm{m}$ and $312 \mathrm{~g} / \mathrm{m}$ ) and its variance (ranging between $1779 \mathrm{~g}^{2} / \mathrm{m}^{2}$ and $4573 \mathrm{~g}^{2} / \mathrm{m}^{2}$ ). Nevertheless, we can rule out that change in the phenotypic distribution played a major role in the fluctuation of selection because the estimate of variation in the slope of selection was almost identical in models where the phenotype was standardized among years versus within years. Fluctuation in selection was therefore the result of variation in the fitness landscape, but we do not know what drove this variation

If we are to gain a deeper understanding of the dynamics of the fitness landscape and the ecological drivers of selection, we ultimately need to move beyond the estimation of variance parameters, toward a more mechanistic understanding of the genetic and ecological sources of phenotypic variation and their covariance with fitness (Morrissey \& Hadfield, 2012). Good examples where we know the detailed ecological driver of variation in selection are still scarce. Some notable exceptions include beak size in Darwin finches (Grant \& Grant, 2002), reproductive timing in great tits (Husby et al., 2011), and insecticide resistance in Culex mosquitoes (Milesi et al., 2016). All of these, as well as the present study, rely on individual-based long-term monitoring, difficult and costly to upkeep, but necessary to disentangle the causes and consequences of selection in natural populations (Clutton-Brock \& Sheldon, 2010). The snow vole monitoring is more complete and spans over more generations (approximately nine) than most longitudinal studies of wild populations, but our simulations highlight that this is not sufficient yet to fully describe the evolutionary consequences of fluctuating selection on size. Future studies might hence consider specifically targeting highly heritable traits (Fig. 5) to obtain a stronger and less variable expected correlation between selection and evolution, while 
they wait for more data to accumulate.

Alternatively, meta-analyses of many replicated estimates of selection may reveal preponderant drivers of selection across species and ecosystems, even if individual studies are often short-term and lack resolution (e.g., Siepielski et al., 2017; Caruso et al., 2017).

\section{Timescale}

Despite fluctuations in the strength and direction of phenotypic selection, the rate and direction of evolution was constant and non-zero over most of the study period. Thereby our findings are at odds with the idea that fluctuating selection causes short-term evolutionary stasis. Nevertheless, fluctuating selection may be a driver of short-term evolutionary dynamics in other natural populations, where the selection measured by regression-based methods is causal and not dominated by an environmental covariation between traits and fitness. Moreover, it is unlikely that fluctuating selection will not be evolutionary relevant on longer time scales, in the snow voles and in other species. Indeed, over geological time scales, bounded fluctuations of phenotypic evolution are increasingly attributed to responses to fluctuating selection, rather than to sampling variation and evolutionary stasis (Uyeda et al., 2011; Voje et al., 2015). Unless the environment is constant, causal selective pressures are likely to change over longer time periods, either because the fitness landscape changes, or because the phenotypic distribution changes through evolutionary adaptation or phenotypic plasticity.

Fluctuating selection and evolution might go undetected because the time frame is too short. For instance in the snow vole population, adaptive evolution and the causal selective pressure causing it are probably related to a short-term climatic anomaly which goes against longterm changes induced by global climate change. On the other hand, we may have missed some fluctuating selection and evolution because the temporal resolution at which selection is estimated is too low, smoothing out very short-term changes in selection and the rate of genetic change. The latter is not unlikely in the snow vole population, where the causal selective pressure varies seasonally: viability selection is null early in the reproductive season and increases throughout summer (Bonnet et al., 2017). 


\section{Conclusion}

While our results do not argue against the evolutionary relevance of fluctuating selection in general, they warn against interpreting any phenotypic fluctuating selection in terms of fluctuating evolution: As the dynamics of selection and evolution can be uncoupled on certain time scales, fluctuating selection does not necessarily provide a general explanation for evolutionary stasis. Thereby we have highlighted the danger of relying on temporally replicated phenotypic estimates of selection to understand and predict the evolutionary dynamics of natural populations. Instead, quantifying the evolutionary relevance of fluctuating selection requires a joined analysis of selection and evolution.

\section{Acknowledgments}

Thanks to two anonymous reviewers for constructive corrections and suggestions. Thanks to Jarrod D. Hadfield, Lukas F. Keller, Marc Kéry and Pirmin Nietlisbach for comments on earlier versions of this work. Thanks to the many field helpers over 11 years. The snow vole monitoring was authorised by the Amt für Lebensmittelsicherheit und Tiergesundheit, Chur, Switzerland, and supported by the Claraz-Donation. This work was funded by Swiss National Science Foundation project grants ... MASKED DURING ANONYMOUS REVIEWING.

\section{References}

Bell, G. 2010. Fluctuating selection: the perpetual renewal of adaptation in variable environments. Philos. Trans. R. Soc. B pp. 87-97.

Bergland, A.O., Behrman, E.L., O'Brien, K.R., Schmidt, P.S. \& Petrov, D.A. 2014. Genomic Evidence of Rapid and Stable Adaptive Oscillations over Seasonal Time Scales in Drosophila. PLoS Genetics 10: e1004775.

Blanckenhorn, W. 2000. The evolution of body size: what keeps organisms small? $Q$. Rev. Biol. 75: 385-407. 
Bonnet, T. \& Postma, E. 2016. Successful by Chance? The Power of Mixed Models and Neutral Simulations for the Detection of Individual Fixed Heterogeneity in Fitness Components. Am. Nat. 187: 60-74.

Bonnet, T., Wandeler, P., Camenisch, G. \& Postma, E. 2017. Bigger Is Fitter? Quantitative Genetic Decomposition of Selection Reveals an Adaptive Evolutionary Decline of Body Mass in a Wild Rodent Population. PLoS Biol. 15: e1002592.

Brookfield, J.F. 2016. Why are estimates of the strength and direction of natural selection from wild populations not congruent with observed rates of phenotypic change? BioEssays 38: 1-8.

Caruso, C.M., Martin, R.A., Sletvold, N., Morrissey, M.B., Wade, M.J., Augustine, K.E., Carlson, S.M., MacColl, A.D.C., Siepielski, A.M. \& Kingsolver, J.G. 2017. What Are the Environmental Determinants of Phenotypic Selection? A Meta-analysis of Experimental Studies. Am. Nat. 190: 363-376.

Chevin, L.M. \& Haller, B.C. 2014. The temporal distribution of directional gradients under selection for an optimum. Evolution 68: 3381-3394.

Chevin, L.M., Visser, M.E. \& Tufto, J. 2015. Estimating the variation, autocorrelation, and environmental sensitivity of phenotypic selection. Evolution 69: 2319-2332.

Clutton-Brock, T. \& Sheldon, B.C. 2010. Individuals and populations: the role of longterm, individual-based studies of animals in ecology and evolutionary biology. Trends Ecol. Evol. 25: 562-573.

Darwin, C. 1859. On the origin of species. London, UK: John Murray.

de Villemereuil, P., Schielzeth, H., Nakagawa, S. \& Morrissey, M. 2016. General methods for evolutionary quantitative genetic inference from generalised mixed models. Genetics 204: $1281-1294$.

Endler, J.A. 1986. Natural selection in the wild. Princeton, NJ: Princeton University Press. 
Estes, S. \& Arnold, S.J. 2007. Resolving the paradox of stasis: models with stabilizing selection explain evolutionary divergence on all timescales. Am. Nat. 169: 227-244.

Firth, J.A., Hadfield, J.D., Santure, A.W., Slate, J. \& Sheldon, B.C. 2015. The influence of nonrandom extra-pair paternity on heritability estimates derived from wild pedigrees. Evolution 69: 1336-1344.

Fisher, R. 1958. The genetical theory of natural selection, 2nd edn. Dover Publications, New York.

Fisher, R.a. \& Ford, E.B. 1947. The spread of a gene in natural conditions in a colony of the moth Panaxia dominula L. Heredity 1: 143-174.

Frank, S.A. 2012. Natural selection. IV. The Price equation. J. Evol. Biol. 25: 1002-1019.

García-Navas, V., Bonnet, T., Waldvogel, D., Wandeler, P., Camenisch, G. \& Postma, E. 2015. Gene flow counteracts the effect of drift in a Swiss population of snow voles fluctuating in size. Biol. Conserv. 191: 168-177.

Grant, P.R. \& Grant, B.R. 2002. Unpredictable evolution in a 30-year study of Darwin's finches. Science 296: 707-711.

Gubbay, J., Collignon, J., Koopman, P., Capel, B., Economou, A., Munsterberg, A. \& al. 1990. A gene mapping to the sex-determining region of the mouse $\mathrm{Y}$ chromosome is a member of a novel family of embryonically expressed genes. Nature 346: 245-250.

Hadfield, J.D. 2010. Mcmc methods for multi-response generalized linear mixed models: The MCMCglmm R package. J. Stat. Soft. 33: 1-22.

Hadfield, J.D., Richardson, D.S. \& Burke, T. 2006. Towards unbiased parentage assignment : combining genetic, behavioural and spatial data in a Bayesian framework. Mol. Ecol. 15: 3715-3730.

Hadfield, J.D., Wilson, A.J., Garant, D., Sheldon, B.C. \& Kruuk, L.E.B. 2010. The misuse of BLUP in ecology and evolution. Am. Nat. 175: 116-25. 
Henderson, C.R. 1950. Estimation of genetic parameters. Ann. Math. Stat. 21: 309-310.

Henderson, C.R. 1975. Best linear unbiased estimation and prediction under a selection model. Biometrics 31: 423-447.

Henderson, C.R. 1976. Simple method for computing inverse of a numerator relationship matrix used in prediction of breeding values. Biometrics 32: 69-83.

Hendry, A.P. 2017. Eco-evolutionary dynamics. Princeton University Press.

Hendry, A.P. \& Kinnison, M.T. 1999. Perspective: the pace of modern life: measuring rates of contemporary microevolution. Evolution 53: 1637-1653.

Hereford, J., Hansen, T. \& Houle, D. 2004. Comparing strengths of directional selection: how strong is strong? Evolution 58: 2133-2143.

Hunt, J., Bussière, L.F., Jennions, M.D. \& Brooks, R. 2004. What is genetic quality? Trends Ecol. Evol. 19: 329-333.

Husby, A., Visser, M.E. \& Kruuk, L.E.B. 2011. Speeding up microevolution: The effects of increasing temperature on selection and genetic variance in a wild bird population. PLoS Biol. 9: e1000585.

Janeau, G. \& Aulagnier, S. 1997. Snow vole - Chionomys nivalis (Martins 1842). IBEX J. of Mountain Ecol. 4: 1-11.

Janzen, F.J. \& Stern, H.S. 1998. Logistic regression for empirical studies of multivariate selection. Evolution 52: 1564-1571.

Jones, A.G., Arnold, S.J. \& Bürger, R. 2004. Evolution and stability of the g-matrix on a landscape with a moving optimum. Evolution 58: 1639-1654.

Jones, O.R. \& Wang, J. 2010. COLONY : a program for parentage and sibship inference from multilocus genotype data. Mol. Ecol. Resour. 10: 551-555.

Kingsolver, J.G. \& Diamond, S.E. 2011. Phenotypic selection in natural populations: what limits directional selection? Am. Nat. 177: 346-57. 
Kingsolver, J.G., Hoekstra, J.M., Berrigan, D., Vignieri, S.N., Hill, C.E., Hoang, A. \& al. 2001. The strength of phenotypic selection in natural populations. Am. Nat. 157: $245-261$

Kruuk, L.E.B. 2004. Estimating genetic parameters in natural populations using the "animal model". Philos. Trans. R. Soc. B 359: 873-90.

Lande, R. 1976. Natural selection and random genetic drift in phenotypic evolution. Evolution 30: 314-334.

Lande, R. 1979. Quantitative Genetic Analysis of Multivariate Evolution , Applied to Brain : Body Size Allometry. Evolution 33: 402-416.

Lande, R. \& Arnold, S.J.. 1983. The Measurement of Selection on Correlated Characters. Evolution 37: 1210-1226.

Luque-larena, J.J., López, P. \& Gosálbez, J. 2002. Microhabitat use by the snow vole Chionomys nivalis in alpine environments reflects rock-dwelling preferences. Can. J. Zool. 80: 36-41.

Merilä, J., Sheldon, B.C. \& Kruuk, L.E.B. 2001. Explaining stasis : microevolutionary studies in natural populations. Genetica 112: 199-222.

Milesi, P., Lenormand, T., Lagneau, C., Weill, M. \& Labbé, P. 2016. Relating fitness to long-term environmental variations in natura. Mol. Ecol. 25: 5483-5499.

Morrissey, M.B. 2015. Evolutionary quantitative genetics of nonlinear developmental systems. Evolution 69: 2050-2066.

Morrissey, M.B. \& Hadfield, J.D. 2012. Directional selection in temporally replicated studies is remarkably consistent. Evolution 66: 435-42.

Morrissey, M.B., Kruuk, L.E.B. \& Wilson, a.J. 2010. The danger of applying the breeder's equation in observational studies of natural populations. J. Evol. Biol. 23: 2277-88. 
Morrissey, M.B., Parker, D.J., Korsten, P., Pemberton, J.M., Kruuk, L.E.B. \& Wilson, A.J. 2012. The prediction of adaptive evolution: empirical application of the secondary theorem of selection and comparison to the breeder's equation. Evolution 66: 23992410.

Morrissey, M.B. \& Wilson, A.J. 2010. PEDANTICS : an R package for pedigree-based genetic simulation and pedigree manipulation, characterization and viewing. Mol. Ecol. Resour. 10: 711-719.

Mousseau, T.A. \& Roff, D.A. 1987. Natural selection and the heritability of fitness components. Heredity 59: 181-197.

Postma, E. 2014. Four decades of estimating heritabilities in wild vertebrate populations: improved methods, more data, better estimates? In: Quantitative genetics in the wild (A. Charmentier, D. Garant \& L.E.B. Kruuk, eds), 1st edn, pp. 16-33. Oxford University Press, Oxford, U.K.

Price, G. 1972. Extension of covariance selection mathematics. Ann. Hum. Gen. 35: $485-490$

Price, G.R. 1970. Selection and covariance. Nature 227: 520-521.

Price, T. \& Liou, L. 1989. Selection on clutch size in birds. Am. Nat. 134: 950-959.

Quaas, R. 1988. Additive genetic model with groups and relationships. J. Dairy Sci. 71: $1338-1345$.

Qvarnström, A., Brommer, J.E. \& Gustafsson, L. 2006. Testing the genetics underlying the co-evolution of mate choice and ornament in the wild. Nature 441: 84-86.

R Core Team 2015. R: A Language and Environment for Statistical Computing. R Foundation for Statistical Computing, Vienna, Austria. URL http://www.R-project.org/.

Rausher, M.D. 1992. The measurement of selection on quantitative traits: biases due to environmental covariances between traits and fitness. Evolution 46: 616-626. 
Reed, T.E., Gienapp, P. \& Visser, M.E. 2016. Testing for biases in selection on avian reproductive traits and partitioning direct and indirect selection using quantitative genetic models. Evolution 70: 2211-2225.

Robertson, A. 1966. A mathematical model of the culling process in dairy cattle. Anim. Prod. 8: 95-108.

Robinson, M.R., Pilkington, J.G., Clutton-Brock, T.H., Pemberton, J.M. \& Kruuk, L.E.B. 2008. Environmental Heterogeneity Generates Fluctuating Selection on a Secondary Sexual Trait. Curr. Biol. 18: 751-757.

Siepielski, A.M., Dibattista, J.D. \& Carlson, S.M. 2009. It's about time : the temporal dynamics of phenotypic selection in the wild. Ecol. Lett. 12: 1261-1276.

Siepielski, A.M., Morrissey, M.B., Buoro, M., Carlson, S.M., Caruso, C.M., Clegg, S.M. \& al. 2017. Precipitation drives global variation in natural selection. Science 355: 959-962.

Smouse, P.E., Meagher, T.R. \& Lobak, C.J. 1999. Parentage analysis in Chaemeclirium luteum (L.) Gray (Liliaceae): why do some males have higher contributions? J. Evol. Biol. 12: 1069-1077.

Stinchcombe, J.R., Agrawal, A.F., Hohenlohe, P.a., Arnold, S.J. \& Blows, M.W. 2008. Estimating nonlinear selection gradients using quadratic regression coefficients: double or nothing? Evolution 62: 2435-40.

Uyeda, J.C., Hansen, T.F., Arnold, S.J. \& Pienaar, J. 2011. The million-year wait for macroevolutionary bursts. Proc. Natl. Acad. Sci. 108: 15908-15913.

Voje, K.L., Holen, Ø.H., Liow, L.H. \& Stenseth, N.C. 2015. The role of biotic forces in driving macroevolution: beyond the Red Queen. Proc. Roy. Soc. B 282: 1-9.

Wade, M.J. 2006. Natural selection. In: Evolutionary genetics: concepts and case studies. (C.W. Fox \& J.B. Wolf, eds), pp. 399-413. Oxford University Press, Oxford, U.K. 
Wandeler, P. \& Camenisch, G. 2011. Identifying Y-chromosomal diversity by longtemplate PCR. Mol. Ecol. Resour. 11: 835-841.

Wandeler, P., Ravaioli, R. \& Bucher, T.B. 2008. Microsatellite DNA markers for the snow vole (Chionomys nivalis). Mol. Ecol. Resour. 8: 637-639.

Wang, J. 2004. Sibship reconstruction from genetic data with typing errors. Genetics 166: $1963-1979$.

Wolak, M.E. \& Reid, J.M. 2017. Accounting for genetic differences among unknown parents in microevolutionary studies: how to include genetic groups in quantitative genetic animal models. J. Anim. Ecol. 86: 7-20.

Wood, S. 2011. Fast stable restricted maximum likelihood and marginal likelihood estimation of semiparametric generalized linear models. J. R. Stat. Soc. 73: 3-36. 
Table 1: Number of phenotyped individuals, survivors to the next year, and number of immigrants.

\begin{tabular}{lrrrrrrrrrrr}
\hline Year & 2006 & 2007 & 2008 & 2009 & 2010 & 2011 & 2012 & 2013 & 2014 & 2015 & 2016 \\
\hline Phenotyped individuals & 183 & 193 & 139 & 163 & 131 & 56 & 66 & 116 & 130 & 118 & 128 \\
Number of adults & 64 & 66 & 62 & 46 & 69 & 36 & 32 & 40 & 52 & 59 & 65 \\
Number of juveniles & 112 & 126 & 75 & 103 & 59 & 15 & 34 & 75 & 77 & 55 & 63 \\
Number of survivors & 43 & 39 & 33 & 48 & 16 & 8 & 21 & 38 & 31 & 20 & - \\
Number of immigrants & 52 & 13 & 13 & 14 & 3 & 9 & 9 & 8 & 11 & 5 & 1 \\
\hline
\end{tabular}

Notes: The number of phenotyped adults and juveniles includes all individuals with at least one body mass index measurement in a given year, i.e. with a measurement of both body mass and body length. This represents the sample size for the selection analyses based on total fitness $(F)$ and viability $(\phi)$. The number of survivors to the next year represents the sample size for the selection analysis based on fertility $(\rho)$, and is still unknown for 2016. Immigrants are individuals with unknown parents, and are counted only in the first year they appeared in the population. In 2006 the number of immigrants represents the size of the base population, while in other years the number of immigrants represents individuals immigrating in the population. 
Table 2: Selection and temporal variation in total selection $(F)$, fertility selection $(\rho)$ and viability selection $(\phi)$ for body mass index.

\begin{tabular}{ccccccc}
\hline Selection & $\beta_{z}(\mathrm{SE})$ & $\mathrm{SD}_{\text {year }}$ & $\overline{\mathrm{SE}_{\text {year }}}$ & $\beta_{z}^{\prime}(\mathrm{SE})$ & $\sigma_{\zeta} 95 \% \mathrm{CI}$ & $\sigma_{\zeta} /\left|\beta_{z}^{\prime}\right|$ \\
\hline Total & $0.639(0.18)$ & 1.198 & 0.753 & $0.598(0.309)$ & $0.691[0.461 ; 1.153]$ & 1.156 \\
Fertility & $-0.204(0.098)$ & 0.277 & 0.160 & $-0.236(0.219)$ & $0.512[0.385 ; 0.779]$ & 2.167 \\
Viability & $0.433(0.126)$ & 0.843 & 0.533 & $0.439(0.252)$ & $0.642[0.409 ; 1.024]$ & 1.462 \\
\hline
\end{tabular}

Notes: $\beta_{z}(S E)$ is the selection gradient across all years and its standard error; $\mathrm{SD}_{\text {year }}$ is the standard deviation of annual selection gradients; $\overline{\mathrm{SE}_{\mathrm{year}}}$ is the mean standard error of these annual estimates; $\beta_{z}^{\prime}(S E)$ is the selection gradient for the average year and its standard error; $\sigma_{\zeta} 95 \% C I$ is the standard deviation of the distribution of selection gradients and its $95 \%$ confidence interval; $\sigma_{\zeta} /\left|\beta_{z}^{\prime}\right|$ is the ratio of the standard deviation in selection over the absolute median year selection, and indicates the likelihood of reversal in the direction of selection. All variables were estimated from generalized linear (mixed) models using standardized body mass index. 
(A)

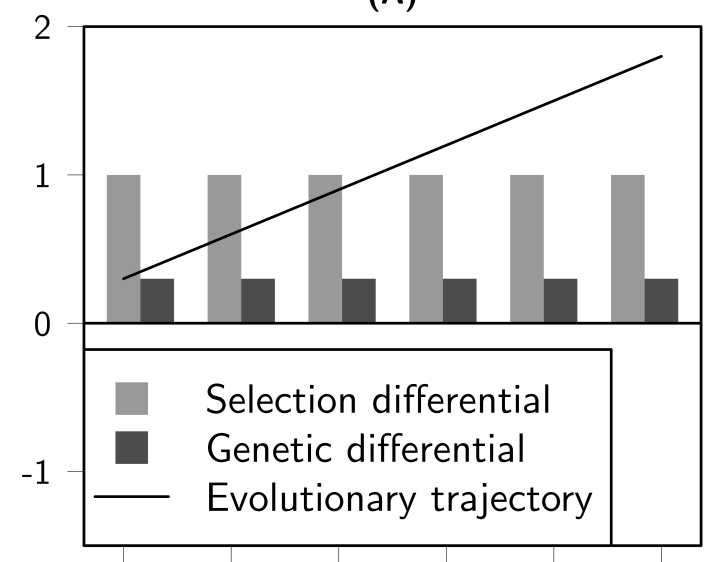

(C)

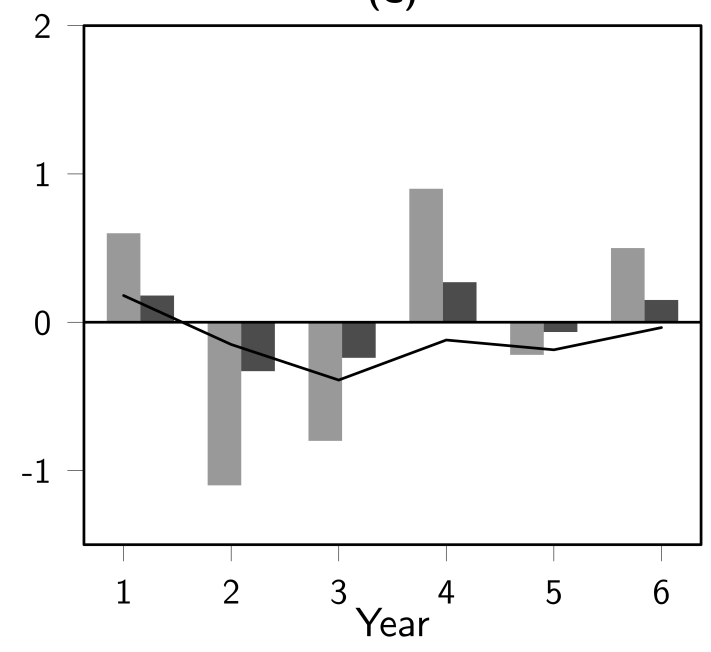

(B)

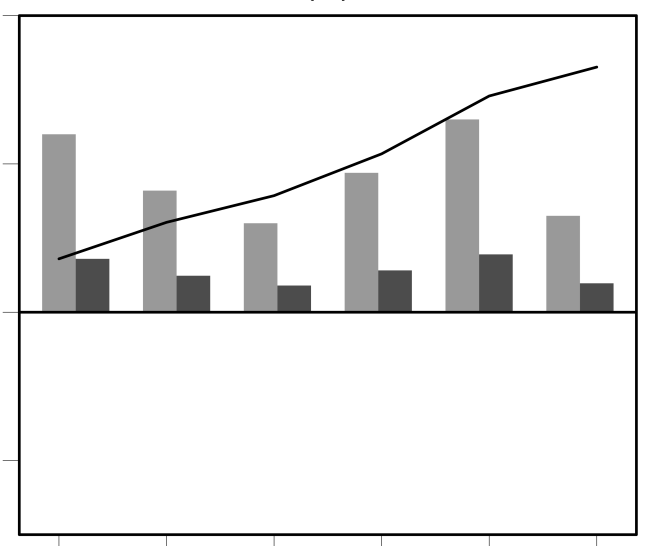

(D)

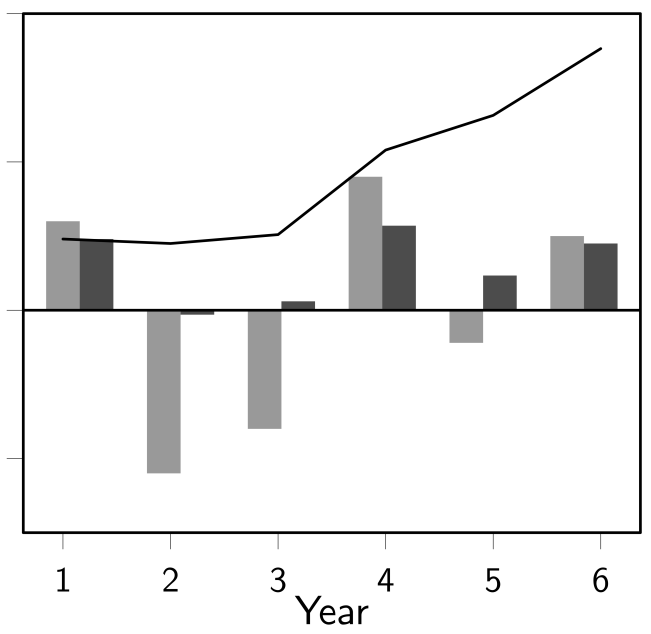

Figure 1: Evolutionary change under constant and fluctuating selection regimes. In (A), selection is constant across years. Following the breeder's equation, the change in breeding values (i.e. genetic differential or the response to selection) is equal to the product of the selection differential and the narrow-sense heritability, which is here set to 0.3. The resultant cumulative response to selection, i.e the evolutionary trajectory, is described by a straight line. In (B), selection fluctuates but does not reverse, and mean selection and the rate of evolution are only slightly reduced compared to (A). In (C), selection fluctuates and reverses, resulting in fluctuating and reversing evolution, and thereby evolutionary stasis over the time frame considered. In (D), selection fluctuates and reverts as in (C), but selection is partly non-causal and mediated by an unobserved environmental factor (i.e. a key assumption of the breeder's equation is violated). As a consequence, selection and evolution are uncoupled and despite fluctuating selection the rate of evolution is similar to (A). 


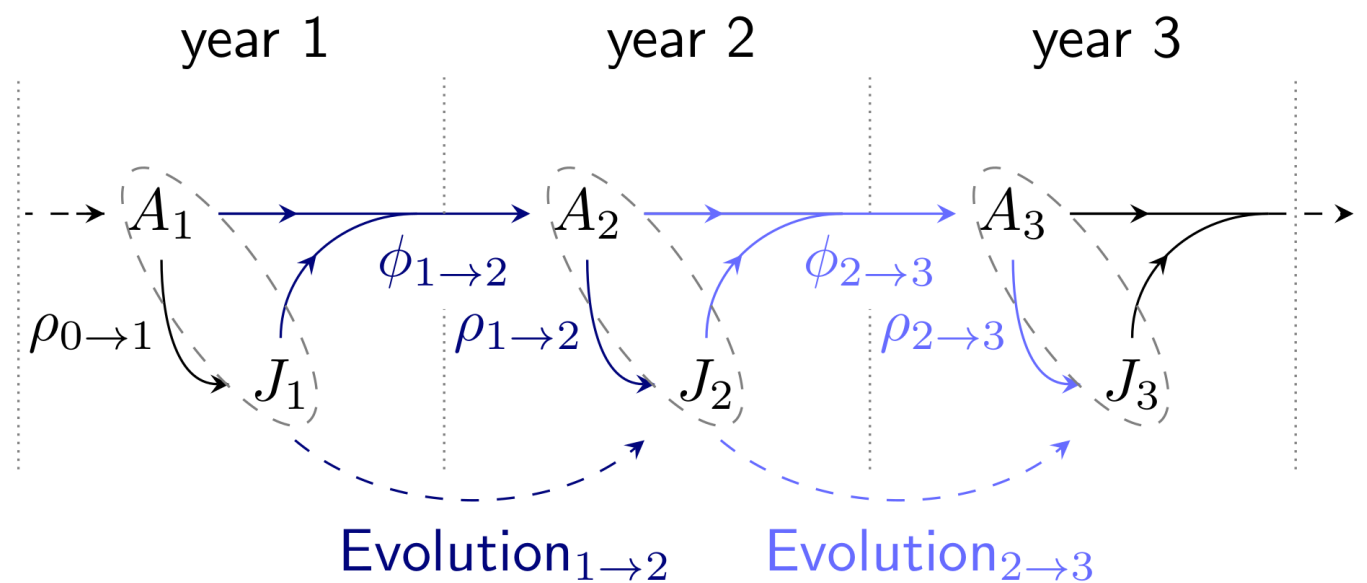

Figure 2: Schematic representation of the snow vole life-cycle, and of our definition of reproduction, survival and and evolution. We are interested in predicting Evolution Evt $_{t \rightarrow t}$, the genetic difference (i.e. the difference in mean breeding value) between all individuals present in year $t$ (adults $\left(A_{t}\right)$ and juveniles $\left(J_{t}\right)$ ) and all individuals present in year $t+1$ $\left(A_{t+1}\right.$ and $\left.J_{t+1}\right)$. This genetic change is a response to viability selection from year $t$ to year $t+1\left(\phi_{t \rightarrow t+1}\right)$ and to fertility selection during year $t+1\left(\rho_{t \rightarrow t+1}\right)$. Three years, and two transitions are depicted. The color (dark blue or light blue) shows which fitness components predict which evolutionary change. 


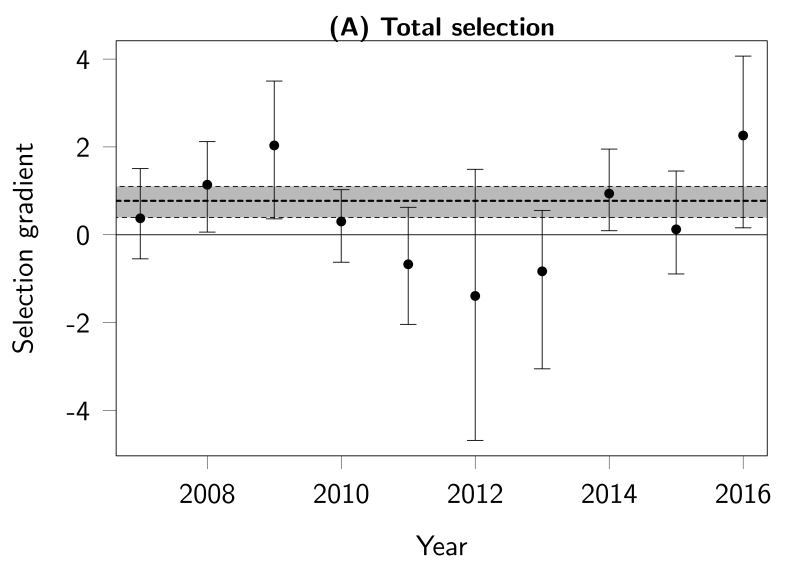

(B) Fertility selection

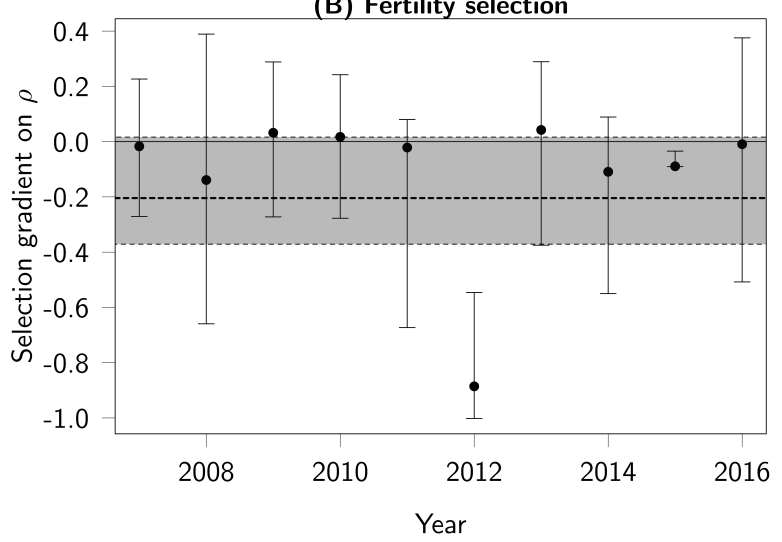

(C) Viability selection

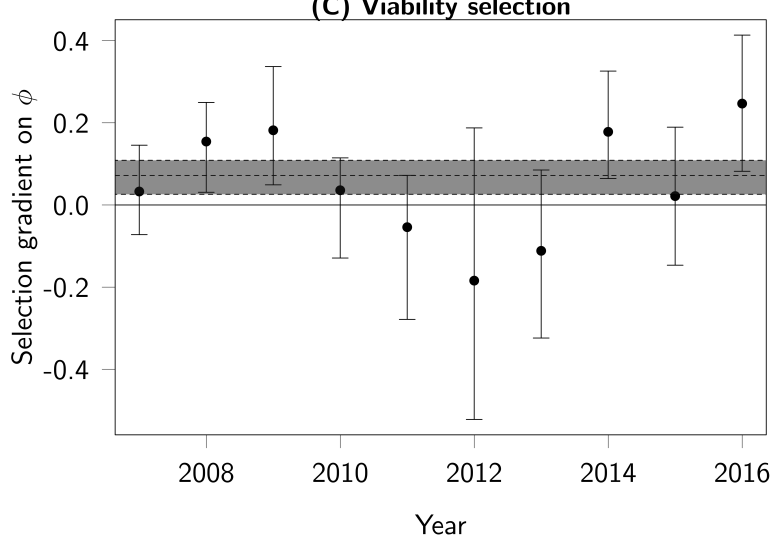

Figure 3: Estimates of (A) total, (B) viability and (C) fertility selection gradients, yearby-year and across all years. Yearly estimates (black dots with 95\%CI error bars) were obtained by fitting separate GLMs for each year. The overall estimate (dashed line with $95 \%$ CI depicted in gray) was produced by pooling all years together. 


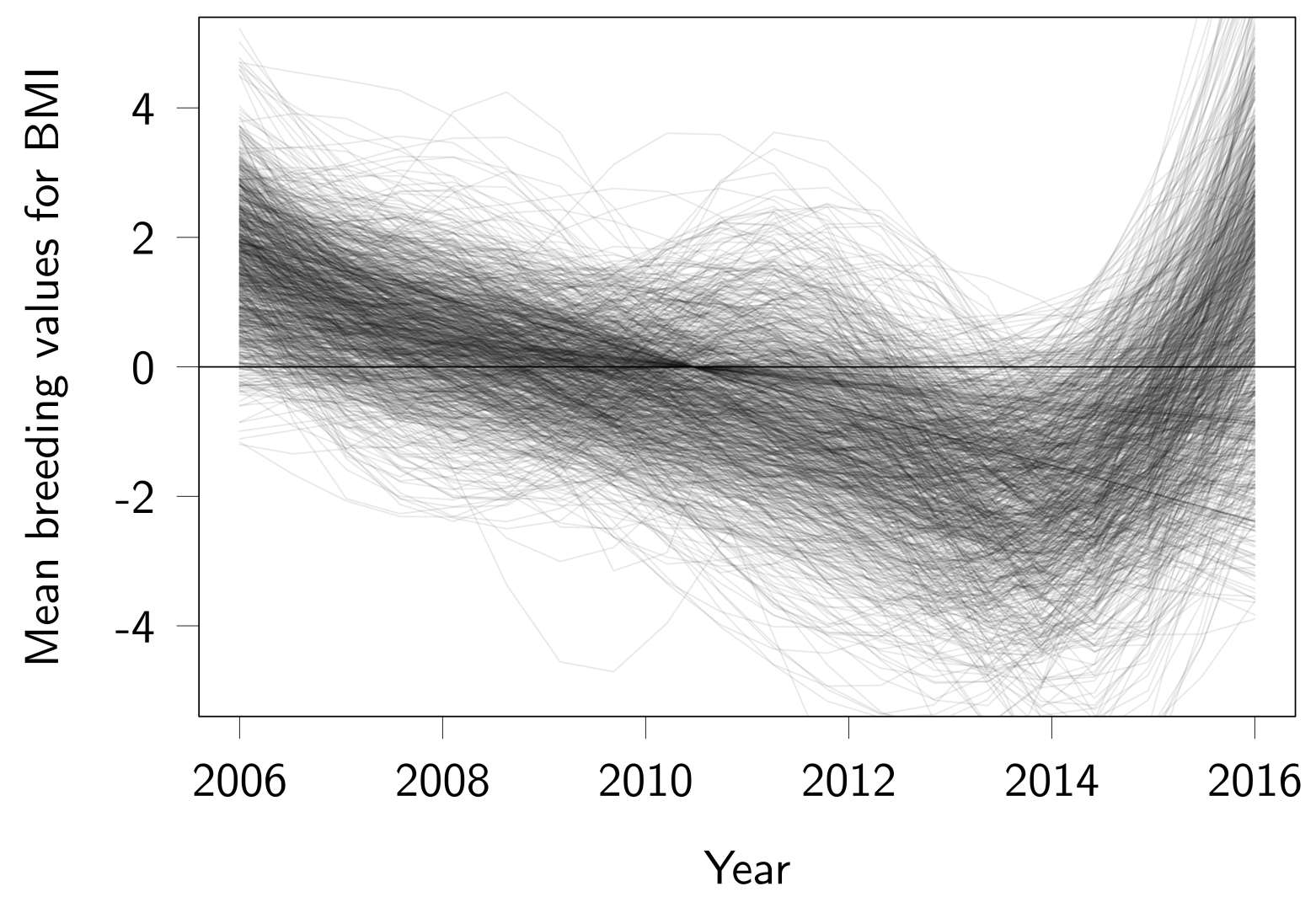

Figure 4: Temporal dynamics of mean breeding values for BMI. Each line was obtained from a different MCMC posterior sample, by fitting a time-spline to the mean of estimated breeding values among individuals alive in any given year. 


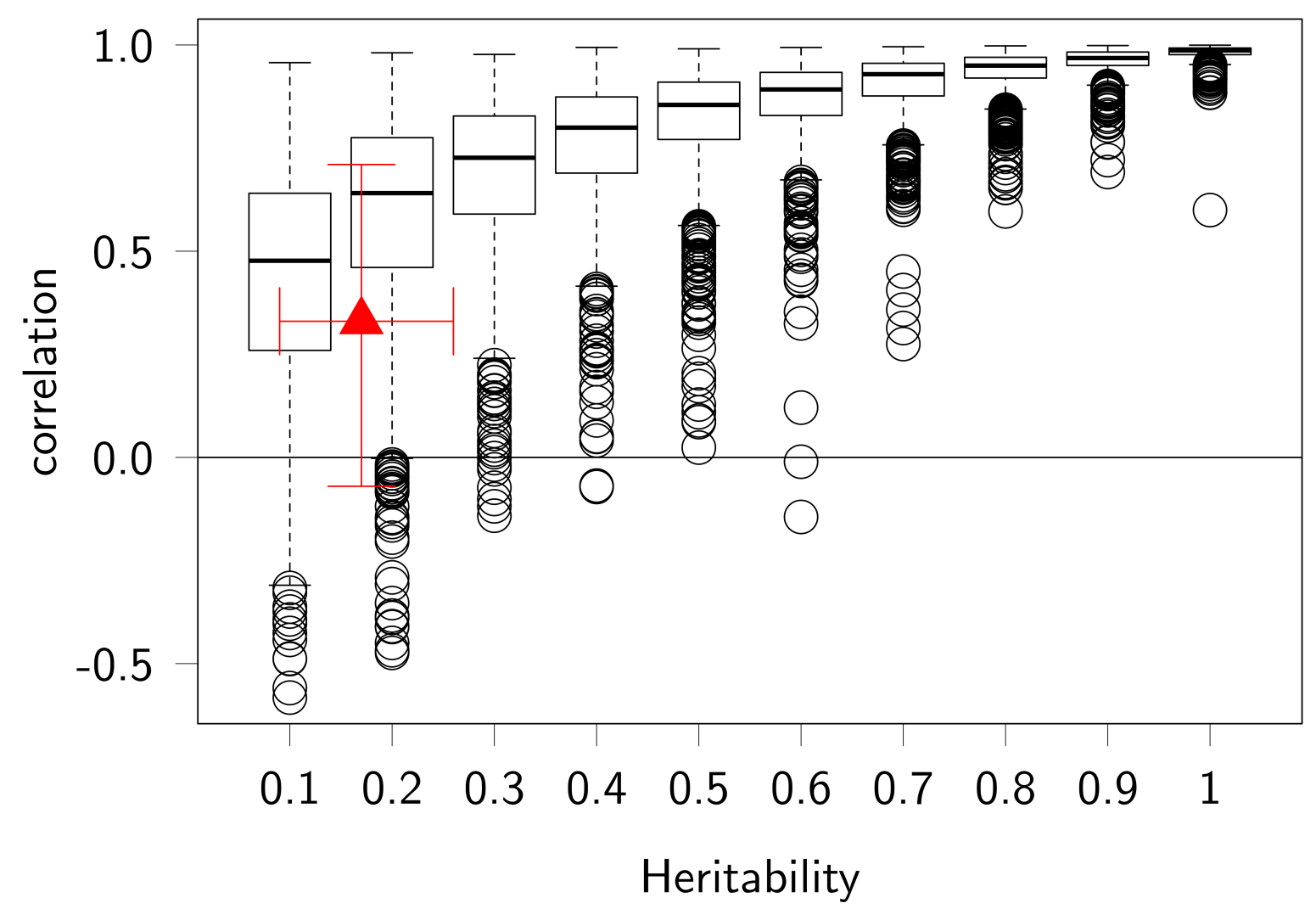

Figure 5: Realized correlation between selection and evolution as a function of simulated heritability. Selection was measured as a standardized selection differential on annualized fitness, and evolution was measured as the difference between mean breeding values of individuals present on one year and those presents on the next year. Simulations consisted of eleven years (as in the snow vole data set). The empirical estimates for the heritability and the correlation are drawn in red, with their confidence intervals. 


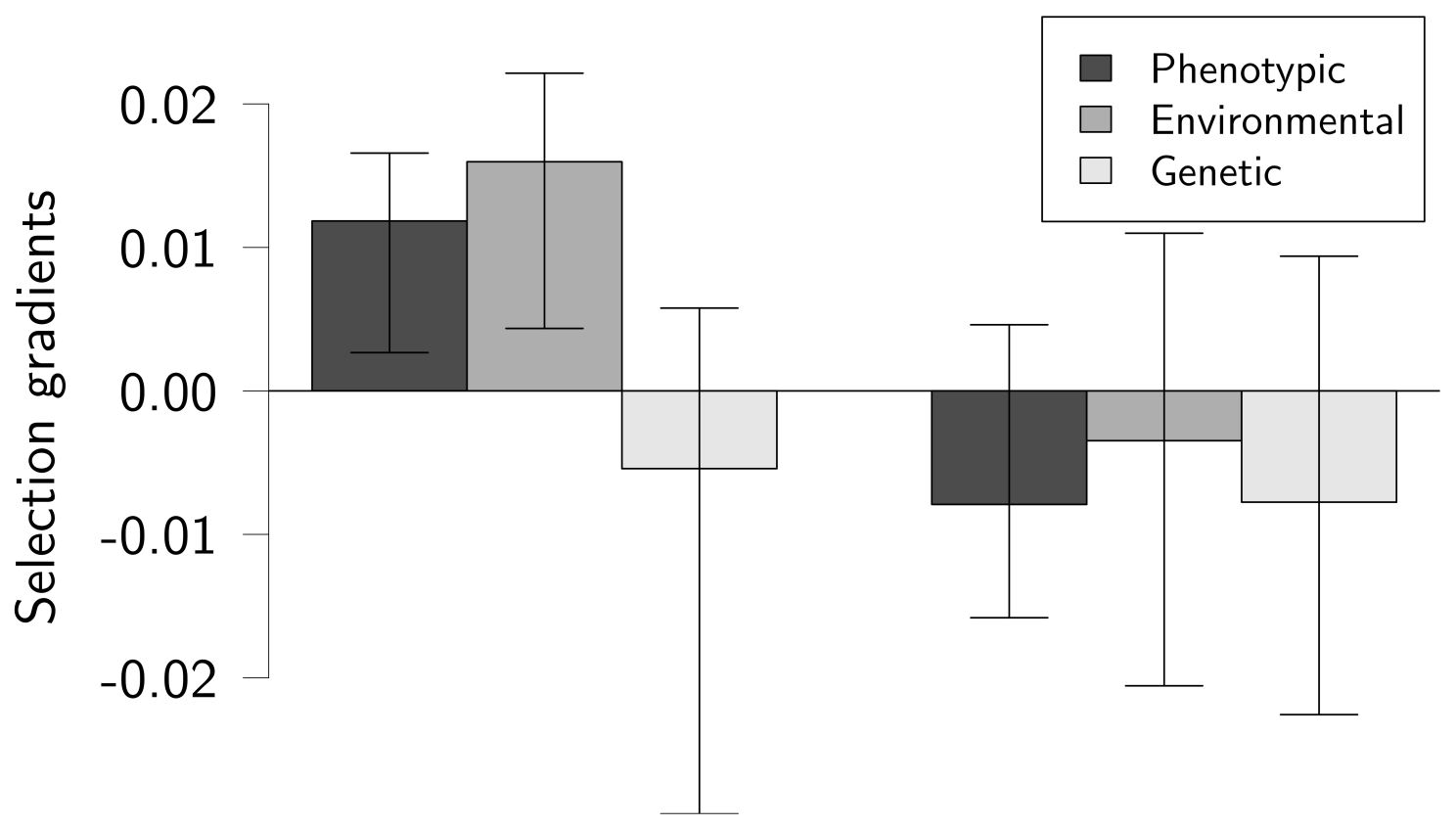

Positive selection years Negative selection years

Figure 6: Phenotypic selection gradients and their decomposition into environmental and genetic gradients for years with positive selection on BMI and for years with negative selection on BMI. Error bars show 95\% confidence intervals. 
Supplementary information for the article Fluctuating selection and its (elusive) evolutionary consequences in a wild rodent population

\section{Contents}

1 Individual-based simulations 1

1.1 Simulation algorithm . . . . . . . . . . . . . . . . 2

1.2 Simulation analyses . . . . . . . . . . . . . . . 3

2 Prior visualization 5

3 Alternative splitting of the dataset $\quad 6$

4 Estimation of the bias introduced by splitting the dataset on the basis of the direction of phenotypic selection

\section{Individual-based simulations}

We used individual-based computer simulations to explore the expected relationship between selection and evolution in a population similar to the snow vole population that is the subject of this study. 


\subsection{Simulation algorithm}

The simulations follow the snow vole life cycle and are parameterized using empirical data for our study population. We simulate a heritable phenotypic trait, as well as reproductive success and survival from one year to the next, in discrete time. The simulated populations are monitored for 11 years, assuming perfect knowledge of individual survival and reproduction during the study period, but their fate beyond this period is unknown.

We consider two age classes: non-reproducing juveniles and reproducing adults. At every time-step (year), a new cohort of $n_{y}$ juveniles appears (where $n_{y}$ is the number of juveniles observed in year $y$ in the real data). Adults get attributed juveniles with a probability depending on their phenotypic trait value. Hence, we simulate "soft selection" (in the sense that selection is all about competition within the population, and phenotypes do not have fixed fitness values), which provides control over the population size. After reproduction, winter comes and imposes some sex- and age-specific mortality. These sex and age differences are estimated from the real data across all years, and do not vary among years. Similarly, an individual's probability of survival depends on its phenotype. Again, this corresponds to soft selection, and ensures that the population size is similar to that of the empirical data. Furthermore, by removing any demographic stochasticity we avoid simulated populations going extinct. Adult survival probability does not vary with age until the fourth year, but all individuals still alive at that point die during the next winter. If they survive their first winter, juveniles recruit to become adults and are able to reproduce.

Viability and fertility selection are uncorrelated and vary from year to year, following Gaussian distributions with a mean and variance parameterized to obtain across-years mean selection $\left(\beta_{F}\right)$ and standard standard deviation in selection $\left(S D_{\text {year }}\right)$ similar to our empirical estimates: $\beta_{F}=0.29$ instead of a real value of 0.28 , and $S D_{\text {year }}=0.31$ instead of 0.45 .

The phenotype follows an infinitesimal quantitative genetic model with constant additive genetic variance. Offspring breeding values are drawn from a Gaussian distribution with a mean given by the average breeding values of the parents and a variance equal to 


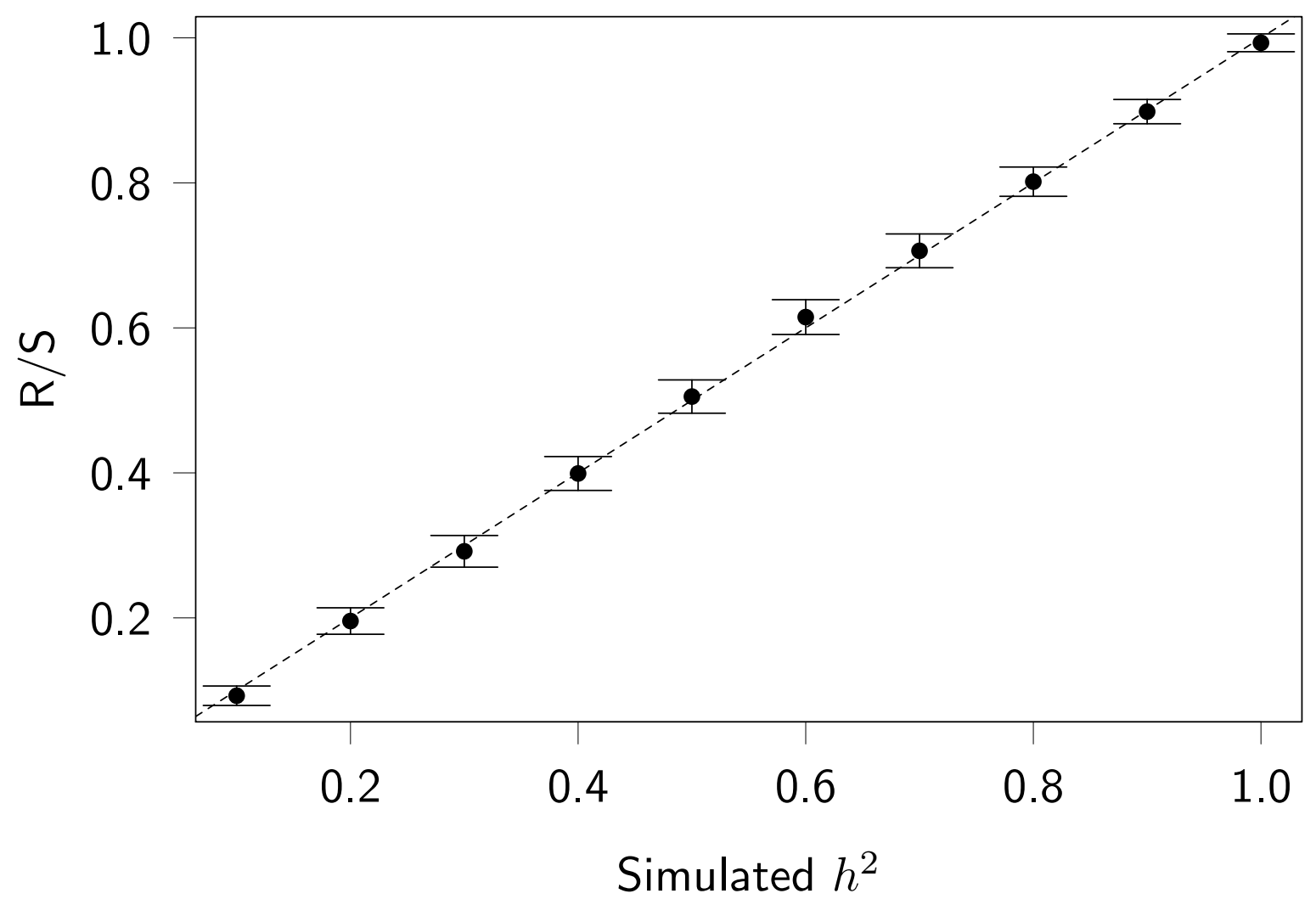

Figure 1.1: Estimated slope of the regression of evolution on selection differential, depending on the simulated heritability. For each value of heritability $\left(h^{2}\right)$, we simulated 100 datasets corresponding each to a eleven-year monitoring of the snow vole population. Error bars represent 95\% confidence intervals. The dashed line is the 1:1 line.

half of additive genetic variance present in the base population.

\subsection{Simulation analyses}

When heritability $\left(h^{2}\right)$ is set to 1 , the selection differential equals the expected change in breeding values $\left(R^{2}=0.99\right)$, and all the variation around this expectation comes from Mendelian segregation (we confirmed this by removing segregation variance from the simulations, which results in $R^{2}=1$ ).

We then estimated the correlation between year-to-year change in breeding values and annual selection gradients, for different values of phenotype heritability and monitoring duration. 


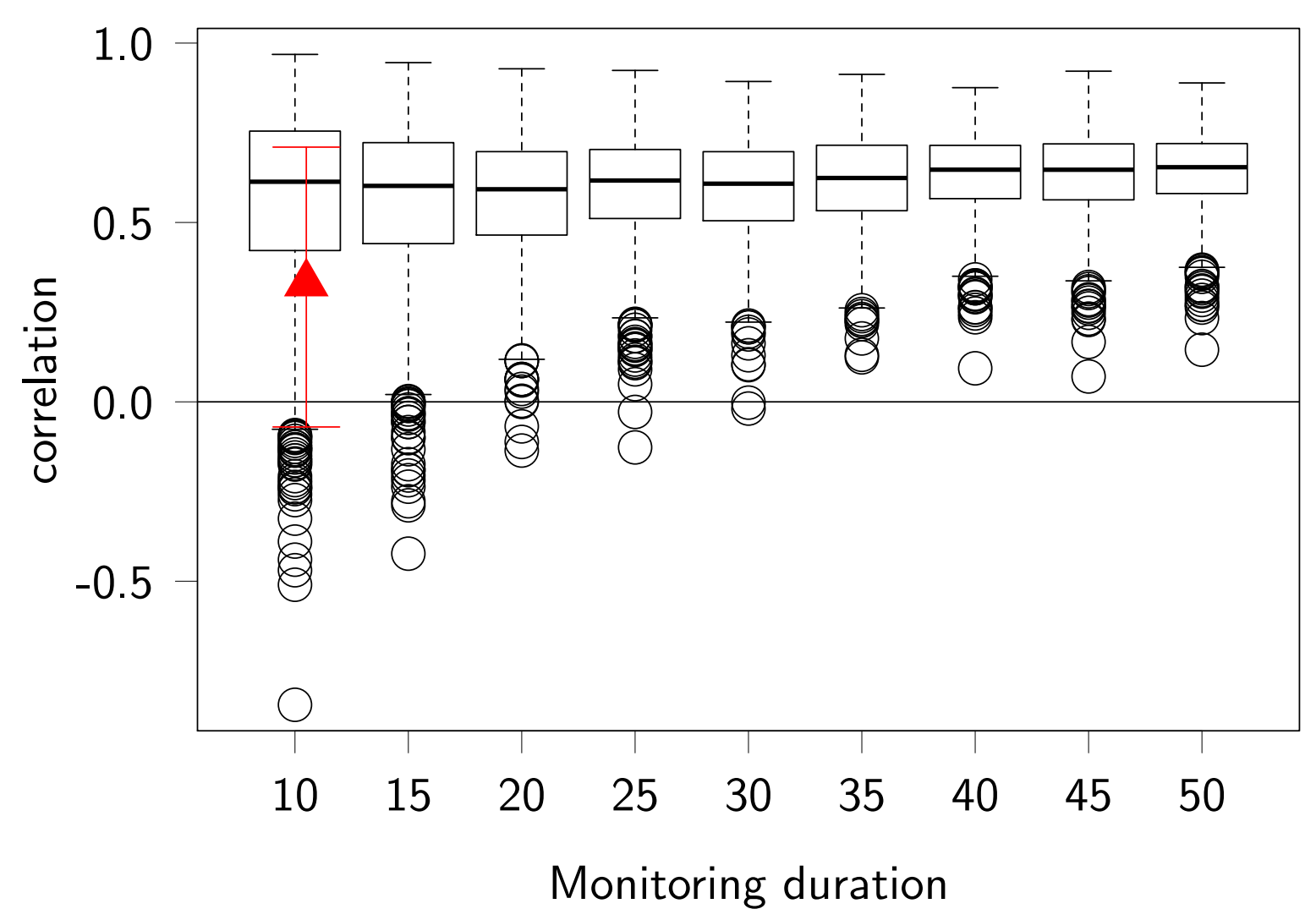

Figure 1.2: Realized correlation between selection and evolution as a function of simulated monitoring length (in black). Heritability was fixed to $17 \%$ (as in the snow vole data set). The empirical correlation estimate is drawn in red, with its confidence interval. 


\section{Prior visualization}
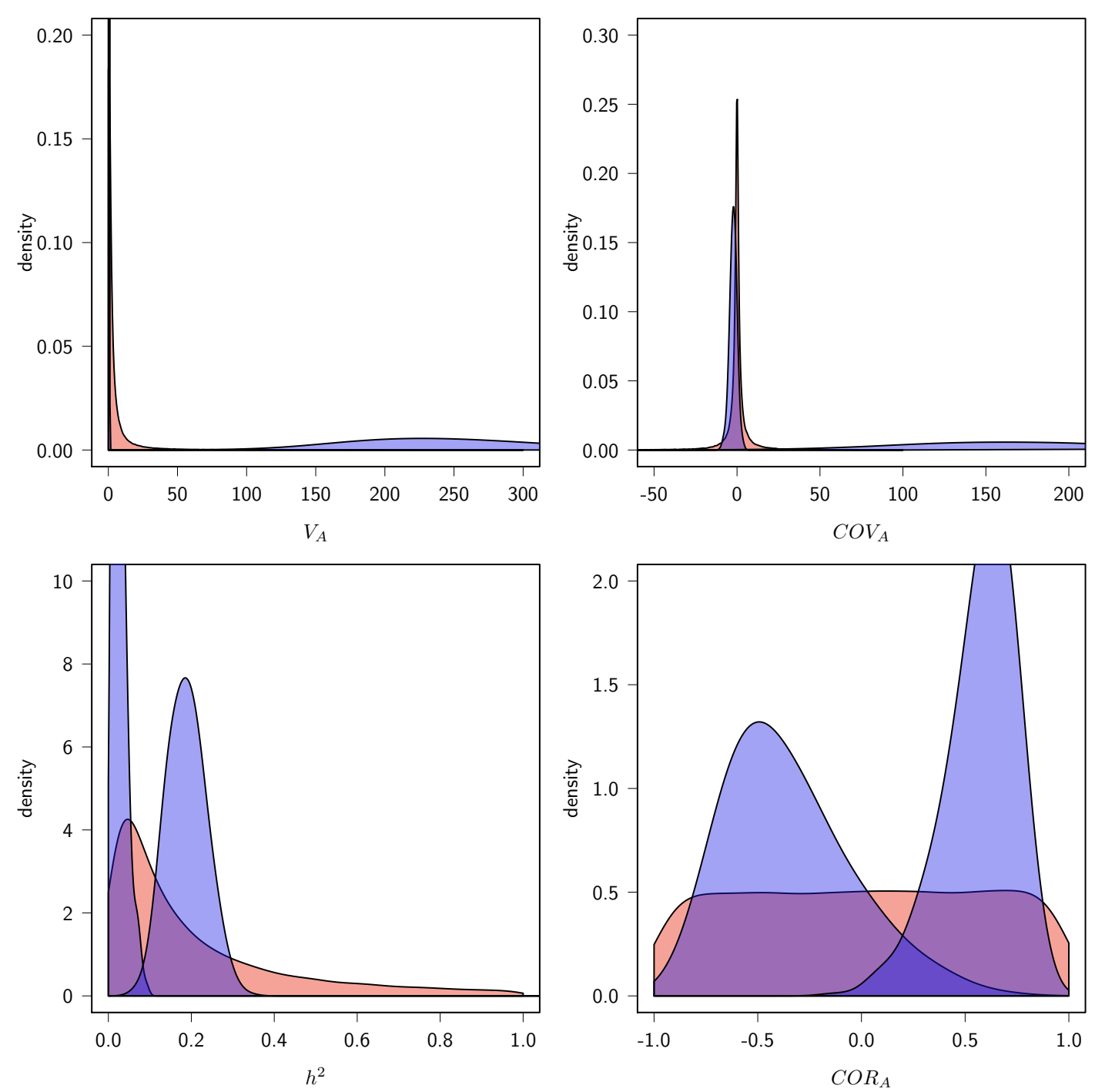

Figure 2.1: Prior and posterior distributions from a trivariate animal model of fitness and body mass index (split in two groups of years, see main text). Red curves represent the prior, blue curves represent the posteriors. The four graphs represent additive genetic variance $\left(V_{A}\right)$, additive genetic covariance $\left(C O V_{A}\right)$, narrow-sense heritability $\left(h^{2}\right)$, and additive genetic correlation $\left(C O R_{A}\right)$. For the sake of clarity, on each graph we show only two posterior densities, out of three existing ones: one involving the fitness trait, and one involving body mass index. 


\section{Alternative splitting of the dataset}

To confirm the stability of the genetic gradients through time, we changed the groups of years (every second year in group 1, other years in group 2) and refitted the tri-variate model. As in the main model, the estimates of genetic gradients were slightly negative and close to zero: first group of years: -0.001 95\% CI [-0.018; 0.017] ; second group of years: $-0.00895 \%$ CI $[-0.022 ; 0.008]$. The difference between the two genetic gradients was again close to zero $(0.00395 \% \mathrm{CI}[-0.008 ; 0.024])$, and the variation between phenotypic gradients $(-0.01295 \%$ CI $[-0.026 ;-0.003])$ was explained by variation in environmental gradients $(-0.02395 \%$ CI $[-0.038 ;-0.006])$. The genetic gradient differed from the environmental gradient in one group of years $(-0.02795 \% \mathrm{CI}[-0.048 ;-0.006])$, but not in the other one $(-0.00195 \% \mathrm{CI}[-0.022 ; 0.022])$. 


\section{Estimation of the bias introduced by splitting the dataset on the basis of the direction of phenotypic selection}

Splitting the dataset into years of positive and years of negative selection may generate a biased estimate of the difference between additive genetic and environmental gradients $\left(\beta_{A}-\beta_{E}\right)$, because the difference is estimated from a model output, rather than explicitly fitted in the model.

To assess the strength and direction of this bias, we simulated 1,000 data sets with no expected differences in the gradients on any year (that is, $\beta_{A}-\beta_{E}=0, \beta_{A+}-\beta_{E+}=0$ and $\left.\beta_{A-}-\beta_{E-}=0\right)$.

To this end, we used the function phensim in the R-package pedantics (Morrissey \& Wilson, 2010) to generate dummy annual fitness BMI values, but using the same structure as the real data, in terms of replication and distribution across years. Although these dummy data have the same expected additive genetic variation and environmental variation as is estimated from the real data, the expected genetic and environmental (and hence phenotypic) covariation between BMI and fitness is zero. We subsequently replaced the real fitness and BMI values for the simulated ones.

Although we did not simulate a covariance between BMI and fitness, due to chance, the realised covariance between BMI and fitness in each year is either positive or negative, and never exactly zero. We used these random fluctuations in the direction of phenotypic selection to split the dummy data into years with a positive selection differential and years with a negative selection differential. We then fitted an animal model to each of these datasets to estimate $\beta_{A}$ and $\beta_{E}$ in years of positive and negative selection and recorded the posterior modes of $\beta_{A+}-\beta_{E+}$ and $\beta_{A_{-}}-\beta_{E--}$. We repeated the simulation and analysis process 1,000 times to estimate the null-distribution of $\beta_{A+}-\beta_{E+}$ and $\beta_{A-}-\beta_{E-}$ (that is, when we know that they should not differ).

These simulations revealed that a bias arises when splitting the data on the basis of the direction of phenotypic selection (Fig. 4.1). However, in this case, the bias is opposite 
to the pattern observed with the real data: the estimated difference $\beta_{A+}-\beta_{E+}$ is negative for real data while the expected bias is positive; whereas $\beta_{A_{-}-} \beta_{E-}$ is zero for real data while the expected bias is negative.

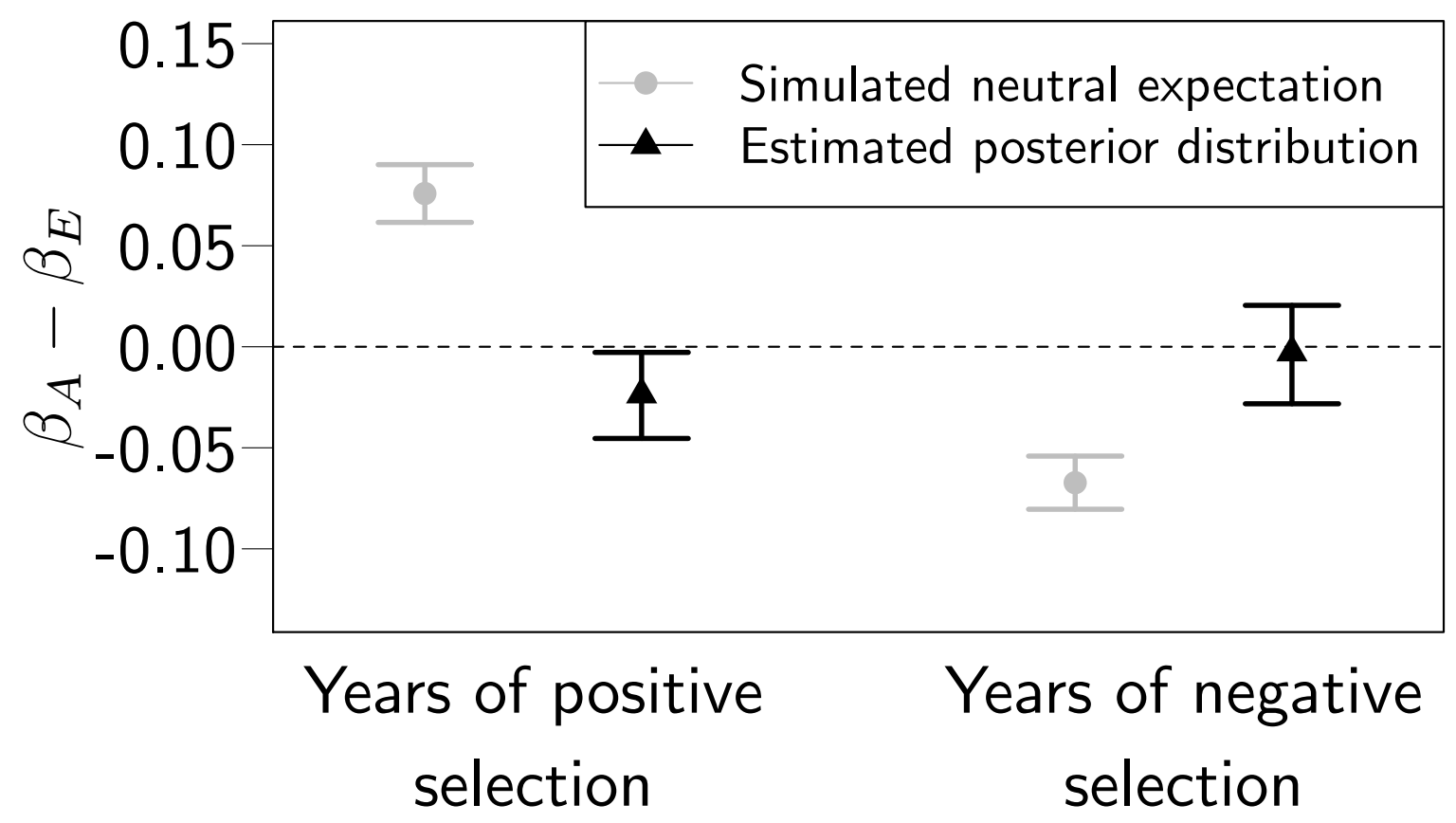

Figure 4.1: Difference between additive genetic gradients and environmental gradients for simulated (gray) and real (black) snow vole data. For the real data (in black), the posterior distribution of the difference was estimated from an animal model of fitness, BMI in years of positive selection, and BMI in years of negative selection (see main text). The simulated neutral expectation shows the bias (mean \pm 2 standard errors, in gray) generated by the split of the data into years of positive selection and years of negative selection. Data were simulated assuming no difference in additive genetic and environmental gradients, and analyzed using animal models of fitness and simulated BMI in years of positive or years of negative selection.

\section{References}

Morrissey, M.B. \& Wilson, A.J. 2010. PEDANTICS : an R package for pedigree-based genetic simulation and pedigree manipulation, characterization and viewing. Mol. Ecol. Resour. 10: 711-719. 